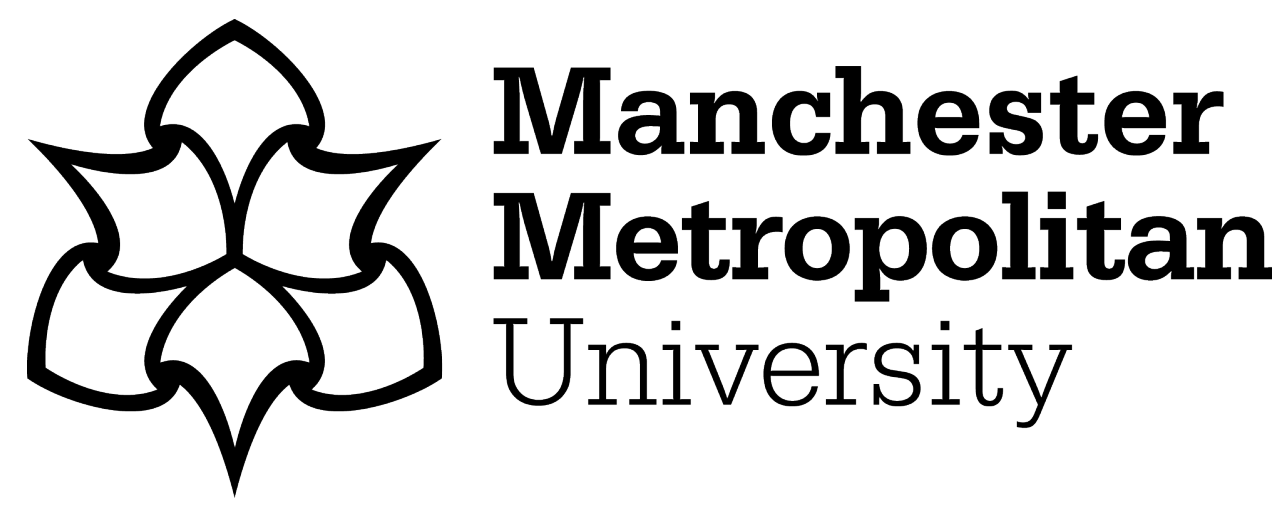

Aichun Feng, Bai, W ORCID logoORCID: https://orcid.org/0000-0002-3537207X, You, Y, Chen, Z-M and Price, WG (2016) A Rankine source method solution of a finite depth, wave-body interaction problem. Journal of Fluids and Structures, 62. pp. 14-32. ISSN 0889-9746

Downloaded from: https://e-space.mmu.ac.uk/625424/

Version: Accepted Version

Publisher: Elsevier

DOI: https://doi.org/10.1016/j.jfluidstructs.2016.01.002

Please cite the published version 


\title{
A Rankine source method solution of a finite depth,wave-body interaction problem
}

\author{
Aichun Feng ${ }^{\mathrm{a}}$, Wei Bai ${ }^{\mathrm{a}}$, Yunxiang You ${ }^{\mathrm{b}}$, Zhi-Min $\mathrm{Chen}^{\mathrm{c}, \mathrm{d}}$, W. G. Price ${ }^{\mathrm{c}, \mathrm{e}}$ \\ ${ }^{a}$ Department of Civil and Environmental Engineering, National University of Singapore, \\ 117576, Singapore \\ ${ }^{b}$ School of Naval Architecture, Ocean and Civil Engineering, Shanghai Jiaotong University, \\ 200030, China \\ ${ }^{c}$ Fluid Structure Interactions Research Group, University of Southampton, SO17 1BJ, UK \\ ${ }^{d}$ School of Mathematics and Statistics, Shenzhen University, Shenzhen 518060, China \\ ${ }^{e}$ WUT-UoS High Performance Ship Technology Joint Centre, Wuhan, 430063, China
}

\begin{abstract}
A Rankine source method is developed adopting continuous free surface and seabed source panel distributions to solve numerically wave-body interaction problems involving various seabed conditions. The free surface and seabed surface profiles are represented by continuous panels rather than a discretization by isolated points. These panels are positioned exactly on the fluid boundary surfaces and the proposed method does not require a desingularization technique. For a two-dimensional forced oscillatory body problem, the influences of a flat, concave or convex/hump shaped seabed at different water depths are investigated. Both heave and sway motions are examined and the accuracy and efficiency of the numerical solutions are validated by comparison with published numerical predictions and, where possible, experimental data. Through these simulated findings, the influences of uneven seabeds on the hydrodynamic characteristics associated with wave-body interaction problems are discussed.
\end{abstract}

Keywords: Rankine source method, continuous source panel, finite water depth, uneven seabeds

\section{Introduction}

In offshore engineering, when the draft of a floating body is not small compared to the water depth, the hydrodynamic influence of finite water depth is important(Van Oortmerssen, 1976) as typified by the evaluation and design of ships and offshore structures operating in near shore and coastal waters (Faltinsen, 1993). However the ocean bottom is usually treated as a flat, solid boundary through which no fluid penetrates.

\footnotetext{
${ }^{*}$ Corresponding Author: Aichun Feng; Email, a.feng@nus.edu.sg
} 
For a flat seabed at uniform water depth, Yu and Ursell (1961) developed a multipole expansion method to examine the behavior of a semi-circular cylinder excited in an oscillatory heave motion. The amplitude of the generated wave showed good agreement with experimental data (Yu and Ursell, 1961). Kim $(1969,1975)$ extended the multipole expansion method to include oscillatory heave, sway and roll motions of the cylinder in water of finite depth. Bai (1977) divided the fluid domain into inner and outer regions. The former is described by finite elements and in the outer region the radiation potential is presented by a set of eigenfunctions with unknown coefficients. These unknown coefficients are determined by matching the boundary condition on the interface of the two regions and by satisfying finite water depth conditions. Hydrodynamic coefficients associated with two-dimensional circular and rectangular cylinders were derived over a wide frequency range with focus on their behaviour at zero frequency. Yeung (1981) further extended this method to investigate a three-dimensional vertical cylinder. Though this method gives a comprehensive theoretical understanding of the finite water depth problem, its capability is restricted to the analysis of simple, symmetric body shapes.

Andersen and He (1985) followed the approach of Bai (1977) but replaced the finite element method with a simple Green function method. This panel method is able to analyse arbitrary shaped bodies and is more numerically efficient than the finite element method. The free surface Green function method associated with deep water can be modified to describe finite water depth, fluidstructure interaction problems. For example, commercial software package, WAMIT, was developed for wave-body interaction problems assuming a flat seabed (Lee, 1995).

A sloped seabed bottom is a more realistic description of a coastal seabed. In this situation, the analysis of floating body motions is more complex than for a flat seabed. The previous described numerical approaches can not be directly applied to changing bottom contours. In the mathematical models developed by Buchner (2006); De Hauteclocque et al. (2009); Ferreira and Newman (2009) a second body is introduced to represent the sloping seabed. The model requires the selection of a specific second body in terms of size and shape which is replicated from case to case, and therefore, it cannot supply a general solution to the problem. To reduce reflections from the sloping seabed, Newman (2012) adopted a matching boundary approach to divide the fluid domain into an interior domain of variable water depth and an exterior domain with constant depth. This curtails the application of the methods to an arbitrary bottom topography.

The Rankine source method coupled to other numerical techniques provides a flexible approach to deal with various boundary condition problems. In general terms the method distributes Rankine sources along free surfaces as well as other fluid domain surfaces to satisfy boundary conditions.

By placing isolated points on the free surface to act as control points and source points away from these control points, the method avoids singularity integrals and only the $\operatorname{lnr}$ (for the two-dimensional case) or $1 / \mathrm{r}$ (for the threedimensional case) term needs inclusion in the integral kernel. Through devel- 
opments this method has become a robust numerical tool to solve various wave and wave-body interaction problems (Cao et al., 1991a; Huang, 1997; Scorpio, 1997; Finn, 2003; Zhang et al., 2010; Bandyk and Beck, 2011).

The adoption of isolated source points to replace the profile of a continuous free surface may cause large numerical discrepancies if they are not properly distributed. Traditionally, the free surface is divided into inner and outer domains (Lee, 1992). A chosen number of source points are evenly positioned in the first 3 wavelength inner domain and exponentially distributed in the outer domain. The number of source points associated to each wavelength is carefully selected to determines the numerical accuracy and efficiency of the chosen numerical scheme of study. The exponential distribution in the outer domain allows a large area of free surface to be covered and therefore long time numerical simulations can be executed without surface wave reflections. This distribution method is discussed extensively in the literature for various wave-body interaction problems (Cao, 1991; Beck et al., 1994; Kring, 1994; Finn, 2003; Lee, 2003; Zhang et al., 2007; Zhang and Beck, 2007; Bandyk, 2009; Zhang et al., 2010)

Previous studies (Schultz et al., 1990; Cao et al., 1991b; Cao, 1991; Lee, 1992; Beck and Scorpio, 1995; Lee, 2003; Zhang and Beck, 2007, 2008; Bandyk, 2009; Zhang et al., 2010; Bandyk and Beck, 2011) show that the discretisation of a continuous free surface profile by isolated control points generates numerical error. Further, the free surface boundary condition is not exactly satisfied on the free surface but only at the source points which are placed above the free surface in the desingularized method causing numerical errors to occur during the numerical simulation.

Recently Feng et al. (2015a, 2014, 2015b) developed a source point distribution method with increasing spaces between points to replace the even space method. This method places a higher density of source points near the floating body and at increasing distance form the body the density of distribution decreases. Through this means, the number of source points is reduced and therefore the computational effort is significantly reduced with numerical accuracy retained or increased. The source points closer to the floating body are of increased strength and of greater numerical influence compared to those located further away. This new source distribution method is further developed as the basis of this study.

The continuous panel method with desingularized technique has been applied to wave-body interaction problems in the deep water condition (Feng et al., 2015a). In that paper numerical comparisons showed better numerical accuracy compared with results obtained by the isolated source point method for both linear and nonlinear problems. Here the free surface and seabed profiles are approximated by a continuous distribution of Rankine source panels, and a numerical method developed in a similar framework to a singularized method. The integral of the Rankine source term lnr on the panel becomes integrable when Rankine source points and control points are located at the same position on a panel. The value of this integral can be derived from a mathematical derivation described by Chen (2012), thus avoiding the desingularized technique.

Both Neumann and Dirichlet boundary conditions are required to be satisfied 
at the intersection point since singularities may arise if not treated properly. In the present method, continuous panels are distributed on both sides of the intersection point. The boundary conditions are satisfied respectively at the centre points of the panels which are positioned separately to the intersection points. By this method, free surface and body boundary conditions are satisfied respectively on neighbouring panels.

In this study, a linear free surface condition is assumed and a third order Adams-Bashforth scheme is adopted in the free surface time stepping iteration process. Section 1 gives background information and literature review for finite depth, wave-body interaction problem. Following this section the bound-

ary integral equation, numerical simulation process and panel distribution are demonstrated in Section 2. In Subsection 3.1 numerical convergence is tested for wave-body interaction problem involving a flat seabed. Extensive comparisons are further provided between derived numerical predictions and other available data to provide a measure of validation of the proposed method for this problem. Numerical simulation is further extended to uneven seabed: concave shaped seabed in Subsection 3.2 and convex or humped shaped seabed in Subsection 3.3. A general discussion is provided for seabed shape and water depth effects on the hydrodynamic characteristics of wave-body interaction problems in Subsection 3.4 before the conclusion part in Section 4.

\section{Problem formulation}

\subsection{Fluid motion equations}

Figure 1 shows a coordinate frame of reference $O x z$ with origin $O$ at the centre of a two dimensional body floating in a fluid domain of finite water depth. The fluid domain $\Omega$ is bounded by a free surface $S_{f}$, body surface $S_{b}$, seabed surface $S_{0}$ and an enclosing surface at infinity, $S_{\infty}$. The body undergoes forced oscillatory heave or sway motion in the direction of the $O z$ or $O x$ axis. For heave motion, the body moves in the $\mathrm{Z}$ axis direction while for sway motion the body moves in the $\mathrm{X}$ axis direction. At time $t$, its position is given by the displacement function

$$
\mathbf{x}_{0}=(0, a \sin \omega t) \quad \text { or } \quad \mathbf{x}_{0}=(a \sin \omega t, 0)
$$

for an amplitude $a$ and frequency $\omega$, with instantaneous velocity:

$$
\mathbf{v}_{\mathbf{b}}=(0, a \omega \cos \omega t) \text { or } \mathbf{v}_{\mathbf{b}}=(a \omega \cos \omega t, 0) .
$$

The fluid flow is assumed irrotational and its descriptive velocity potential $\phi$ is subject to the Laplace equation

$$
\frac{\partial^{2} \phi}{\partial x^{2}}+\frac{\partial^{2} \phi}{\partial z^{2}}=0 \text { in the fluid domain } \Omega .
$$

The associated kinematic body boundary condition is given by

$$
\frac{\partial \phi}{\partial \mathbf{n}_{b}}=\mathbf{v}_{b} \cdot \mathbf{n}_{b} \text { on } S_{b},
$$


where $\mathbf{n}_{b}$ is the unit normal vector on the body surface pointing into the fluid domain.

The linear kinematic and dynamic free surface boundary conditions are given respectively by

$$
\frac{\partial \eta}{\partial t}-\frac{\partial \phi}{\partial z}=0 \text { on } S_{f}
$$

and

$$
\frac{\partial \phi}{\partial t}+g \eta=0 \text { on } S_{f}
$$

where $\eta$ denotes the wave elevation and $g$ is the gravitational acceleration.

The boundary condition applied on the far field boundary at infinity requires that the disturbance vanishes such that:

$$
\nabla \phi \rightarrow 0 \text { on } S_{\infty} .
$$

It is assumed that no flow penetrates the solid seabed boundary and therefore

$$
\frac{\partial \phi}{\partial n_{0}}=0 \text { on } S_{0}
$$

where $n_{0}$ is the unit normal vector on the seabed surface pointing into the fluid domain.

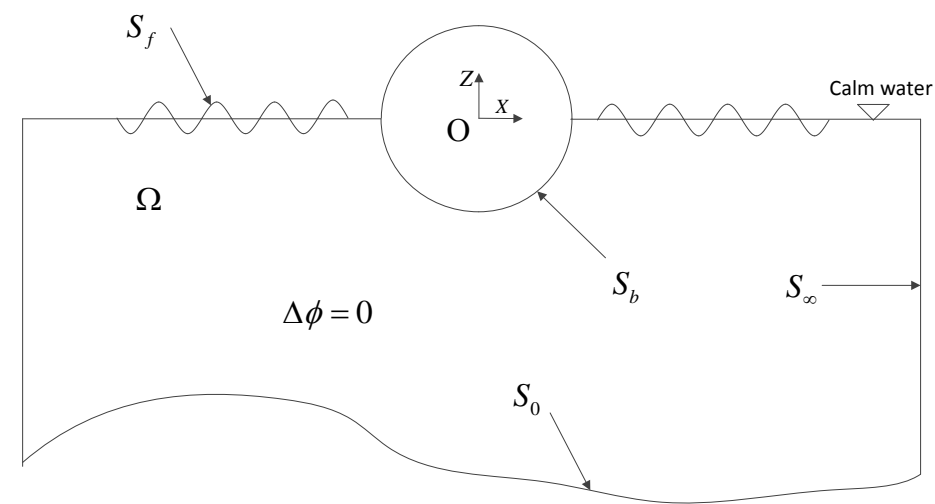

Figure 1: Illustrative sketch of body-fluid interaction problem and definition of coordinate system.

By utilizing the Gauss divergence theorem, Laplace equation (3) and the application of the Dirichlet boundary value problem to the exterior domain of 
the fluid field, Lamb (1945) expresses the velocity potential $\phi$ as a boundary integral of Rankine sources continuously distributed on the fluid boundary $S=$ $S_{b} \cup S_{f} \cup S_{0}$ in the form

$$
\phi\left(\mathbf{x}^{\prime}, t\right)=\int_{S_{b}} \sigma^{b} \ln \left|\mathbf{x}^{\prime}-\mathbf{x}\right| d s_{\mathbf{x}}+\int_{S_{f}} \sigma^{f} \ln \left|\mathbf{x}^{\prime}-\mathbf{x}\right| d s_{\mathbf{x}}+\int_{S_{0}} \sigma^{0} \ln \left|\mathbf{x}^{\prime}-\mathbf{x}\right| d s_{\mathbf{x}} .
$$

Here we denote control point $\mathbf{x}^{\prime}=\left(x^{\prime}, z^{\prime}\right)$ which is located on the middle point of a continuous panel, source point $\mathbf{x}=(x, z)$, the body source strength $\sigma^{b}=$ $\sigma\left(\mathbf{x}^{\prime}, t\right)$, free surface source strength $\sigma^{f}=\sigma\left(\mathbf{x}^{\prime}, t\right)$, seabed source strength $\sigma^{0}=$ $\sigma\left(\mathbf{x}^{\prime}, t\right)$ and variable of integration $d s_{\mathbf{x}}$.

\subsection{Numerical simulation process}

In the current simulation, the fluid domain surface $S$ is discretized by panel Rankine sources rather than isolated Rankine source points. These panel sources are located exactly on the fluid domain surface $S$ and therefore no desingularized technique is applied. Let the integral surfaces $S_{b}, S_{f}$ and $S_{0}$ be approximated as sums of panels $N_{b}, N_{f}$ and $N_{0}$ respectively such as $S_{b}=\cup_{i=1}^{N_{b}} S_{i}^{b}, S_{f}=\cup_{j=1}^{N_{f}} S_{j}^{f}$ and $S_{0}=\cup_{k=1}^{N_{0}} S_{k}^{0}$.

Therefore, the discretization of equation (9) is given by

$$
\begin{aligned}
\phi\left(\mathbf{x}^{\prime}, t\right) & =\sum_{i=1}^{N^{b}} \sigma_{i}^{b} \int_{S_{i}^{b}} \ln \left|\mathbf{x}^{\prime}-\mathbf{x}_{i}\right| d s_{\mathbf{x}} \\
& +\sum_{j=1}^{N^{f}} \sigma_{j}^{f} \int_{S_{j}^{f}} \ln \left|\mathbf{x}^{\prime}-\mathbf{x}_{j}\right| d s_{\mathbf{x}}+\sum_{k=1}^{N^{0}} \sigma_{k}^{0} \int_{S_{k}^{0}} \ln \left|\mathbf{x}^{\prime}-\mathbf{x}_{k}\right| d s_{\mathbf{x}} .
\end{aligned}
$$

Let $\mathbf{x}_{i}^{\prime b} \in S_{b}, \mathbf{x}^{\prime f}{ }_{j} \in S_{f}$ and ${\mathbf{x}^{\prime}}_{k} \in S_{0}$ be control points for $i=1, \ldots, N^{b}$, $j=1, \ldots, N^{f}$ and $k=1, \ldots, N^{0}$. The time iteration scheme starts from the initial conditions applied to the body surface, the free surface and at the seabed, respectively at time $t=0$ given by

$$
\begin{aligned}
& \left.\mathbf{v}_{\mathbf{b}}\left(\mathbf{x}_{i}^{\prime b}, t\right)\right|_{t=0}=(0, a \omega) \text { or }(a \omega, 0) \\
& \left.\phi\left(\mathbf{x}_{j}^{\prime f}, t\right)\right|_{t=0}=0 \text { and }\left.\eta\left(\mathbf{x}_{j}^{\prime}, t\right)\right|_{t=0}=0 \\
& \left.v_{0}\left(\mathbf{x}^{\prime}{ }_{k}, t\right)\right|_{t=0}=0
\end{aligned}
$$

With the use of the body and seabed boundary conditions (4) and (8) respectively and the boundary integral equation (10) with its normal derivative, 
we solve the source strengths $\sigma_{i}^{b}, \sigma_{j}^{f}$ and $\sigma_{k}^{0}$ on $S_{i}^{b}, S_{j}^{f}, S_{k}^{0}$ from the following equations

$$
\begin{aligned}
\phi\left(\mathbf{x}_{j}^{\prime f}, t\right) & =\sum_{i=1}^{N^{b}} \sigma_{i}^{b} \int_{S_{i}^{b}} \ln \left|\mathbf{x}_{j}^{\prime f}-\mathbf{x}_{i}\right| d s_{\mathbf{x}} \\
& +\sum_{j=1}^{N^{f}} \sigma_{j}^{f} \int_{S_{j}^{f}} \ln \left|\mathbf{x}_{j}^{\prime f}-\mathbf{x}_{j}\right| d s_{\mathbf{x}}+\sum_{k=1}^{N^{0}} \sigma_{k}^{0} \int_{S_{k}^{0}} \ln \left|\mathbf{x}_{j}^{\prime f}-\mathbf{x}_{k}\right| d s_{\mathbf{x}}, \\
v_{b n}\left(\mathbf{x}_{i}^{\prime b}, t\right)= & \sum_{i=1}^{N^{b}} \sigma_{i}^{b} \frac{\partial}{\partial n_{b}^{i}} \int_{S_{i}^{b}} \ln \left|{\mathbf{x}^{\prime}}_{i}^{b}-\mathbf{x}_{i}\right| d s_{\mathbf{x}} \\
& +\sum_{j=1}^{N^{f}} \sigma_{j}^{f} \frac{\partial}{\partial n_{b}^{i}} \int_{S_{j}^{f}} \ln \left|\mathbf{x}_{i}^{\prime b}-\mathbf{x}_{j}\right| d s_{\mathbf{x}}+\sum_{k=1}^{N^{0}} \sigma_{k}^{0} \frac{\partial}{\partial n_{b}^{i}} \int_{S_{k}^{0}} \ln \left|\mathbf{x}_{i}^{\prime b}-\mathbf{x}_{k}\right| d s_{\mathbf{x}} \\
0= & \sum_{i=1}^{N^{b}} \sigma_{i}^{b} \frac{\partial}{\partial n_{0}^{k}} \int_{S_{i}^{b}} \ln \left|\mathbf{x}^{\prime 0}-\mathbf{x}_{i}\right| d s_{\mathbf{x}} \\
+ & \sum_{j=1}^{N^{f}} \sigma_{j}^{f} \frac{\partial}{\partial n_{0}^{k}} \int_{S_{j}^{f}} \ln \left|\mathbf{x}^{\prime 0}{ }_{k}-\mathbf{x}_{j}\right| d s_{\mathbf{x}}+\sum_{k=1}^{N^{0}} \sigma_{k}^{0} \frac{\partial}{\partial n_{0}^{k}} \int_{S_{k}^{0}} \ln \left|\mathbf{x}_{k}^{\prime 0}-\mathbf{x}_{k}\right| d s_{\mathbf{x}} .
\end{aligned}
$$

Here $v_{b n}\left(\mathbf{x}_{i}^{\prime b}, t\right)=\mathbf{n}_{b}^{i} \cdot \mathbf{v}_{\mathbf{b}}\left(\mathbf{x}_{i}^{\prime b}, t\right)$ represents the normal velocity on the panels on the body surface $S_{i}^{b}$. $\mathbf{n}_{b}^{i}$ and $\mathbf{n}_{0}^{k}$ denote the unit normal vectors on the panels $S_{i}^{b}$ and $S_{k}^{0}$ respectively.

Equation (14) can be formulated in the following convenient format:

$$
\left[\begin{array}{lll}
C_{N_{f} N_{f}} & C_{N_{f} N_{b}} & C_{N_{f} N_{0}} \\
C_{N_{b} N_{f}} & C_{N_{b} N_{b}} & C_{N_{b} N_{0}} \\
C_{N_{0} N_{f}} & C_{N_{0} N_{b}} & C_{N_{0} N_{0}}
\end{array}\right]\left[\begin{array}{c}
\sigma^{f} \\
\sigma^{b} \\
\sigma^{0}
\end{array}\right]=\left[\begin{array}{c}
\phi\left(\mathbf{x}_{j}^{\prime f}, t\right) \\
v_{b n} \\
0
\end{array}\right],
$$

where $C_{m n}\left(m=N_{f}, N_{b}, N_{0}\right.$ and $\left.n=N_{f}, N_{b}, N_{0}\right)$ are influence coefficient blocks. They reflect the influence of a source panel $n$ on the control point of panel $m$.

The substitution of the source strength values $\sigma_{i}^{b}, \sigma_{j}^{f}$ and $\sigma_{k}^{0}$ at time $t$ obtained from the set of equations (14) produces calculation of the normal velocity $v_{f z}\left(\mathbf{x}_{j}^{\prime f}, t\right)=\frac{\partial \phi\left(\mathbf{x}_{j}^{\prime f}, t\right)}{\partial z}$ on the free surface as

$$
\begin{aligned}
v_{f z}\left(\mathbf{x}_{j}^{\prime f}, t\right) & =\sum_{i=1}^{N^{b}} \sigma_{i}^{b} \frac{\partial}{\partial z} \int_{S_{i}^{b}} \ln \left|\mathbf{x}_{j}^{\prime f}-\mathbf{x}_{i}\right| d s_{\mathbf{x}} \\
& +\sum_{j=1}^{N^{f}} \sigma_{j}^{f} \frac{\partial}{\partial z} \int_{S_{j}^{b}} \ln \left|\mathbf{x}_{j}^{\prime f}-\mathbf{x}_{j}\right| d s_{\mathbf{x}}+\sum_{k=1}^{N^{0}} \sigma_{k}^{0} \frac{\partial}{\partial z} \int_{S_{k}^{0}} \ln \left|\mathbf{x}_{j}^{\prime f}-\mathbf{x}_{k}\right| d s_{\mathbf{x}} .
\end{aligned}
$$


At any time $t$ during the numerical simulation, the body and seabed boundary conditions are described by equations (4) and (8). The free surface conditions are updated according to equations (5) and (6) by a 3rd-order AdamsBashforth scheme, starting from $t=0$, through expressions

$$
\begin{aligned}
& \eta(\Delta t)=v_{f z}(0) \Delta t, \\
& \phi(\Delta t)=-g \eta(\Delta t) \Delta t,
\end{aligned}
$$

and in a 2nd-order Adam-Bashforth scheme, we have

$$
\begin{aligned}
& \eta(2 \Delta t)=\eta(\Delta t)+\frac{\Delta t}{2}\left[3\left(v_{f z}(\Delta t)-v_{f z}(0)\right],\right. \\
& \phi(2 \Delta t)=\phi(\Delta t)+\frac{\Delta t}{2}[-3 g \eta(\Delta t)+g \eta(0)],
\end{aligned}
$$

When the 3rd-order Adam-Bashforth scheme is adopted, we have

$$
\begin{aligned}
& \eta(t+\Delta t)=\eta(t)+\frac{\Delta t}{12}\left[23 v_{f z}(t)-16 v_{f z}(t-\Delta t)+5 v_{f z}(t-2 \Delta t)\right], \\
& \phi(t+\Delta t)=\phi(t)+\frac{\Delta t}{12}[-23 g \eta(t)+16 g \eta(t-\Delta t)-5 g \eta(t-2 \Delta t)] .
\end{aligned}
$$

With strengths $\sigma_{i}^{b}, \sigma_{j}^{f}$ and $\sigma_{k}^{0}$ determined, the velocity potentials on the body surface $S_{b}$ are derived from equation (9) in the form:

$$
\begin{aligned}
\phi\left(\mathbf{x}_{i}^{\prime b}, t\right) & =\sum_{i=1}^{N^{b}} \sigma_{j}^{b} \int_{S_{i}^{b}} \ln \mid \mathbf{x}_{i}^{\prime b} \\
& -\mathbf{x}_{i}\left|d s_{\mathbf{x}}+\sum_{j=1}^{N^{f}} \sigma_{j}^{f} \int_{S_{j}^{f}} \ln \right|{\mathbf{x}^{\prime}}_{i}^{b}-\mathbf{x}_{j}\left|d s_{\mathbf{x}}+\sum_{k=1}^{N^{0}} \sigma_{k}^{0} \int_{S_{k}^{0}} \ln \right|{\mathbf{x}^{\prime}}_{i}^{b}-\mathbf{x}_{k} \mid d s_{\mathbf{x}}
\end{aligned}
$$

The dynamic pressure on the body surface, $p=p\left(\mathbf{x}_{i}^{b}, t\right)$, is evaluated from Bernoulli's equation expressed as

$$
\frac{p(t)}{\rho}=-\frac{\partial \phi}{\partial t}
$$

where $\rho$ denotes the fluid density. The temporal derivative of the potential is calculated by a finite difference method using the the values of the velocity potential at two consecutive time steps.

The dynamic force $F(t)$ applied to the body surface is calculated by integrating the dynamic pressure over the body surface as given by

$$
\mathrm{F}(\mathrm{t})=\int_{S_{b}} p(t) n_{b} d s
$$


By adopting Fourier transforms, the added mass $A_{k k}(\omega)(k=2$, for sway motion and $k=3$ for heave motion) and damping coefficient $B_{k k}(\omega)$ can be obtained as:

$$
\begin{aligned}
& A_{k k}(\omega)=\frac{2}{a \omega^{2} T_{0}} \int_{0}^{T_{0}} F_{k k}(t) \sin (\omega t) d t, \\
& B_{k k}(\omega)=-\frac{2}{a \omega T_{0}} \int_{0}^{T_{0}} F_{k k}(t) \cos (\omega t) d t,
\end{aligned}
$$

where $T_{0}$ denotes the period of the body's motion.

\subsection{Fluid domain panel distribution}

The fluid domain surface is composed of a body surface $S_{b}$, free surface $S_{f}$ and seabed surface $S_{0}$. These surfaces are approximated by continuous panels instead of isolated source points. The integral of the Rankine source term $\ln$ is analytically calculated over the whole panel rather than the algebraic approximation used in traditional isolated source point methods. This change improves numerical accuracy and because it is an analytical integration and carried out over the whole panel, we define this procedure paper as using a continuous Rankine source panel. Figure 2 shows the surface panel distribution associated with an oscillating body in water depth $H$ with a fixed, solid seabed.

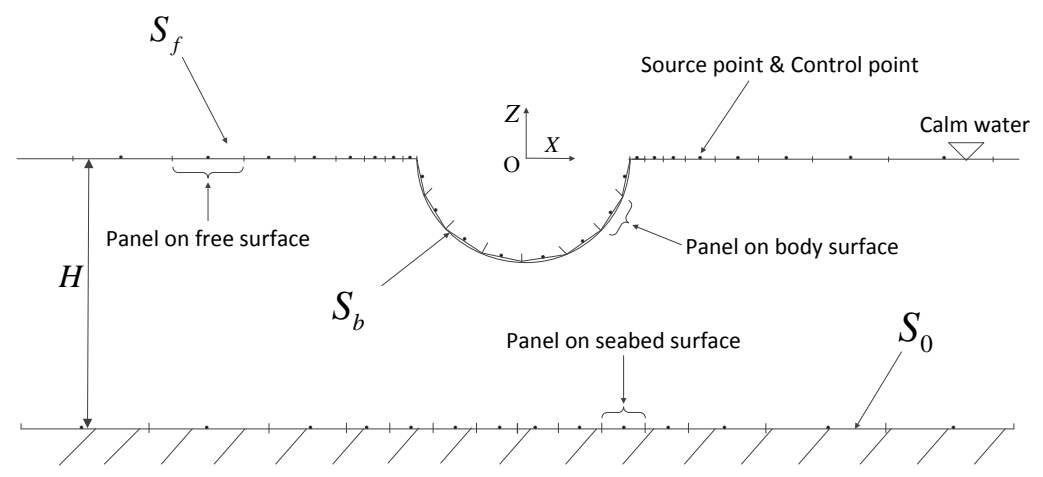

Figure 2: Illustration of panel distribution for oscillating body in finite water depth above a solid seabed.

In the developed mathematical model the body surface is approximated by 40 panels evenly distributed as discussed by Feng et al. (2015a). The free surface is divided into inner and outer domains. To approximate the free surface in the 
inner domain, the source panel distribution method discussed by Feng et al. (2015a, 2014) is adopted. For vertical body motions, the wave-body problem is treated symmetrically with the left hand side free surface sources, arranged as a mirror image of the right hand side. For horizontal body motions, both right and left hand free surface source descriptions are needed. The general theory developed herein only requires minor modifications to treat either vertical or horizontal body motions. For example, the right hand side of $S_{f}$ is divided into inner and outer domains distributed with source grid points $\mathbf{x}_{j}^{\prime}{ }_{j}$. The horizontal distances between neighbouring free surface control (source) points are expressed in the form:

$$
\begin{aligned}
& \left|\mathbf{x}_{j}^{\prime f}-\mathbf{x}_{j-1}^{\prime f}\right|=\frac{L_{b} \alpha_{j}}{\omega^{2}} \text { in the inner domain } \\
& \left|\mathbf{x}_{j}^{\prime f}-\mathbf{x}_{j-1}^{\prime f}\right|=\frac{L_{b} \alpha_{j}}{\omega^{2}} 1.05^{j(j-1) / 2} \text { in the outer domain, }
\end{aligned}
$$

where $L_{b}$ is the length of the body surface panel covering the intersection point of the body and free surface. The parameters $\alpha_{j}$ define the separation distances and are decided according to the method discussed by Feng (2014) which for this reason, is omitted herein. In this distribution scheme, from the intersection points outwards, the importance of the source panel points decreases as the distance increases contrasting with the traditional even distribution method. The first source value is of largest magnitude with the remaining source strengths decaying to zero around the 20th point as discussed by Feng et al. (2015a, 2014).

In the outer domain as indicated by equation (29), the distances between the centres of neighbouring source panels are displaced in an exponentially increasing manner. This allows the encompassment of a very large free surface and therefore the generated wave can move towards the far field of this enlarged computational domain without experiencing wave reflections. By this simple and efficient numerical technique, the radiation condition is well satisfied without the introduction of artificial damping.

The seabed surface is also divided into inner and outer domains. The inner domain is approximated by similar sized panels which simplifies the formulation relating to an uneven seabed surface. The outer domain is arranged similarly to the free surface.

\section{Numerical results}

Numerical simulations are performed using a circular cylinder with a radius $(R)$ to draft $(T)$, ratio $R / T=1$ and a rectangular cylinder with a beam $(B)$ to draft $(T)$, ratio $B / T=2$. In both cases, the amplitude of the oscillating body is $a=0.1 T$. The free surface boundary condition is satisfied on the calm water surface and the body boundary condition is satisfied on the mean wetted body surface because of the chosen small amplitude oscillatory motion. At $t=0$, the gravity and buoyancy forces equate. The floating body is disturbed either by a vertical upward velocity to excite heave motion or a horizontal velocity to excite sway motion. 
Table 1: Numerical convergence of sway damping coefficient $B_{22}$ with different panel numbers on one side of the free surface.

\begin{tabular}{ccc}
\hline Panel no. & Damping coefficient & Relative error \\
\hline 20 & 0.5123 & $16.5 \%$ \\
30 & 0.5746 & $6.34 \%$ \\
40 & 0.6011 & $2.02 \%$ \\
50 & 0.6050 & $1.39 \%$ \\
60 & 0.6078 & $0.93 \%$ \\
70 & 0.6078 & $0.93 \%$ \\
Experimental data of Vugts (1968) & 0.6135 & $0 \%$ \\
Numerical data of Zhang and Beck (2007) & 0.5862 & $4.45 \%$ \\
\hline
\end{tabular}

\subsection{Wave-body interaction problem involving a flat seabed}

In this section, the wave-body interaction problem in constant finite water depth $H=c$ is investigated. The flat seabed condition is approximated by a distribution of continuous panels as shown in the illustration depicted in Figure 2 .

In order to demonstrate the numerical convergence of the proposed method, Numerical simulations were performed for a circular cylinder experiencing forced sway motion in deep water condition. Table 1 shows the numerical convergence of the sway damping coefficient $B_{22}$ at frequency $\omega^{2} B / 2 g=0.5$ with different panel numbers distributed on one side of the free surface. Adopting the experimental data of (Vugts, 1968) as a baseline, a comparison between different predicted data set shows that the relative errors of the current method using 40 - 70 panels are much smaller than the predictions derived from a numerical method adopting desingularized isolated source points and much finer meshes as described by (Zhang and Beck, 2007).

Fluid structure interaction studies were performed for a circular cylinder forced into heave motion in infinite water depth. As the seabed influence behaves in an exponential manner, the seabed produced littel to no effect on the hydrodynamic characteristics in water depth $H \geq 5 R$. For this reason, in this paper, a water depth $H=5 R$ is deemed as an infinite water depth. Figure 3 shows predicted heave added mass $A_{33}$ and damping coefficient $B_{33}$ evaluated by the proposed method compared with the experimental results of Vugts (1968) and numerical results of Frank (1967) adopting a free surface Green function model. The results of the current method show favorable agreement with these sets of published data. For water depths $H=2.0 R$ and $H=1.5 R$, the seabed imposes a significant influence on the wave-body interaction problem. Figure 4 for $H=2.0 R$, and Figure 5 for $H=1.5 R$ show comparisons of predictions of heave added mass $A_{33}$ and damping coefficient $B_{33}$ by the proposed method with the numerical results of Bai (1977) and Andersen and He (1985). All the predictions show good agreement with one another, even though different 
numerical schemes of study are used.

To validate and illustrate the range of the proposed method, this cylinder is forced into sway motion. Figure 6 presents numerical predictions of sway added mass coefficient $A_{22}$ and sway damping coefficient $B_{22}$. These are compared with the experimental data measured by Vugts (1968) and the numerical findings of Frank (1967) for water depth $\mathrm{H}=\infty$. Although the numerical predictions show good agreement, discrepancies are observed between these data sets. It is noted that in the experiments of Vugts (1968) that measurement inaccuracies occurred in the force sensor and there existed a lack of structural stiffness in the experimental instrument package.

Calculated hydrodynamic coefficients and numerical results derived by Andersen and He (1985) for water depth $H=2.0 R$ are shown in Figure 7. This comparison shows very favourable agreement.

Numerical simulations are executed for the rectangular cylinder undergoing forced heave motion to demonstrate the applicability of the proposed algorithm. Figure 8 presents a comparison of heave coefficient $A_{33}$ and $B_{33}$ between current numerical predictions, the experimental data of Vugts (1968), and the numerical results of Zhang and Beck (2007) for a deep water condition. Zhang and Beck (2007) adopted a desingularized Rankine source method with an even distribution of isolated source points on the free surface.

For finite water depth, $H=2.0 R$ above a flat seabed, these two hydrodynamic coefficients are shown in Figure 9 and compared with results from other numerical methods presented by Bai (1977), Yeung (1973) and Rhee (1977). For water depth $H=1.5 R$, Figure 10 presents a comparison of data for these two hydrodynamic coefficients determined by the current method and from the mathematical model developed by Bai (1977). All these comparisons demonstrate the accuracy and range of applicability of the proposed method when dealing with wave-body interaction problems with infinite and finite water depths. These extensive comparisons show good agreement with published data and provide a measure of validation of the proposed method.

\subsection{Wave-body interaction problem in finite water depth above a concave shaped seabed}

In this section, a floating body undergoing forced oscillatory motion above an uneven seabed is numerically investigated based on the proposed method. Concave and convex shaped undulations are two basic seabed topographies ( $\mathrm{Su}$ et al., 2008) which widely exist in the marine environment. For example, the sediment scour or the buried pipeline can significantly change the seabed topography. When the marine structure is located above an undulating seabed surface, the hydrodynamic characteristics associated with the structure can be affected. In the present paper, concave semi-ellipse and convex semi-ellipse shaped seabed topographies are used to represent uneven seabed and their in-

fluence on hydrodynamic characteristics and free surface disturbances are now discussed.

Figure 11 illustrates a concave semi-ellipse shaped seabed beneath the floating body. The major and minor axes of the semi-ellipse are $L m a j=2.0 R$ and 


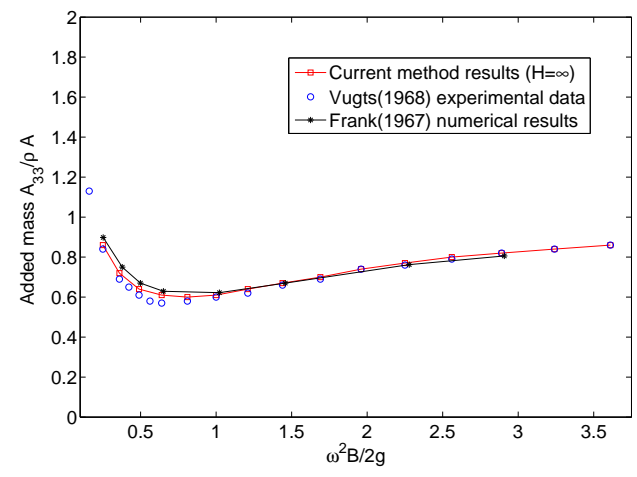

(a) Heave added mass coefficient $A_{33}$

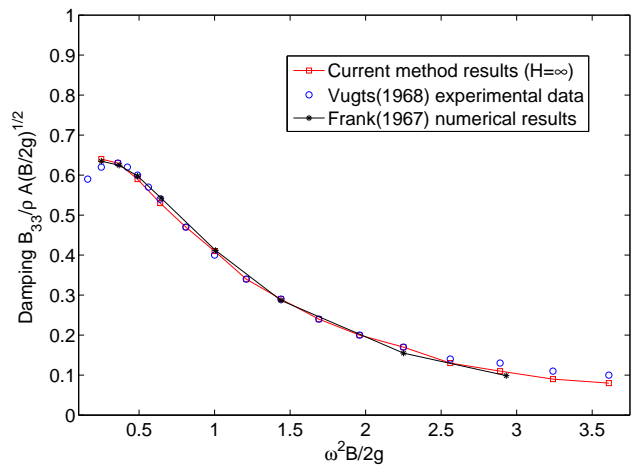

(b) Heave damping coefficient $B_{33}$

Figure 3: Comparison of the predicted heave added mass coefficient $A_{33}$ (a) and damping coefficient $B_{33}$ (b) between the current method, the experimental data of Vugts (1968) and numerical results of Frank (1967) for a circular cylinder oscillating in heave motion in infinite water depth $H=\infty$.

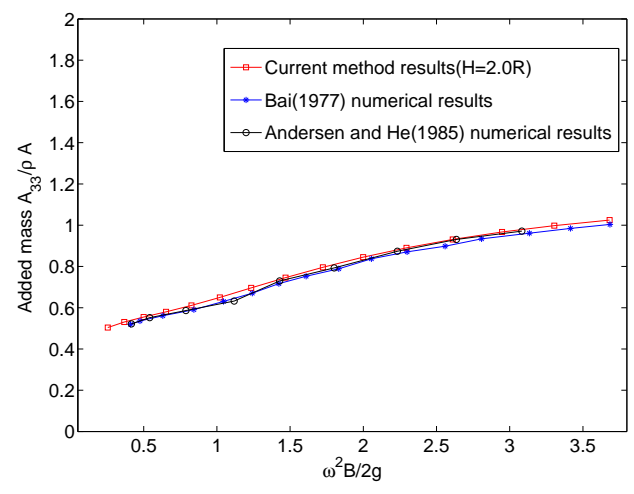

(a) Heave added mass coefficient $A_{33}$

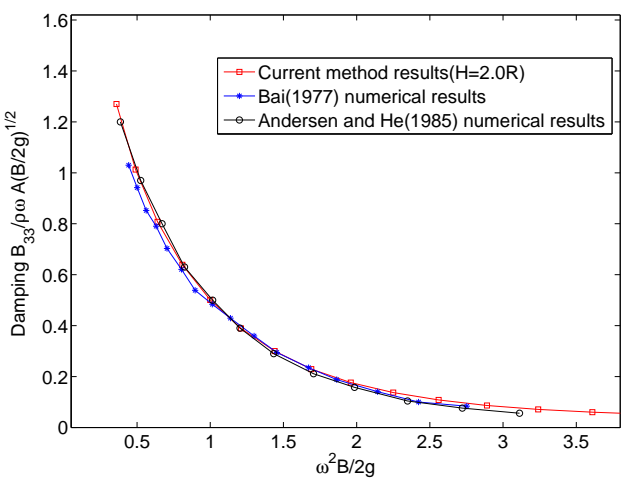

(b) Heave damping coefficient $B_{33}$

Figure 4: Comparison of the predicted heave added mass coefficient $A_{33}$ (a) and damping coefficient $B_{33}$ (b) between the current method, the numerical results of Andersen and He (1985) and Bai (1977) for a circular cylinder oscillating in heave motion in water depth $H=$ $2.0 R$.

Lmin $=0.1 R$, Lmin $=0.2 R$ and $\operatorname{Lmin}=0.3 R$ respectively, each denoted as seabed configuration $(a),(b)$ and $(c)$. For simplicity, the uneven shape retains a measure of symmetry with maximum and mean water depth beneath the floating body increasing from $(a)$ to $(c)$.

Figures 12 and 13 show a comparison of added mass and damping coefficients $A_{33}$ and $B_{33}$ for the circular cylinder experiencing forced heave oscillatory motion above a flat seabed at water depth, $H=1.5 R$, and the uneven surfaces $(a),(b)$ and $(c)$. It is observed that both the added mass coefficient $A_{33}$ and 


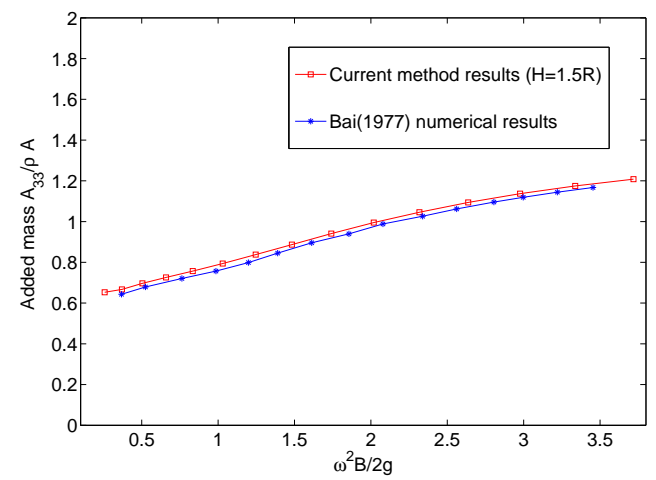

(a) Heave added mass coefficient $A_{33}$

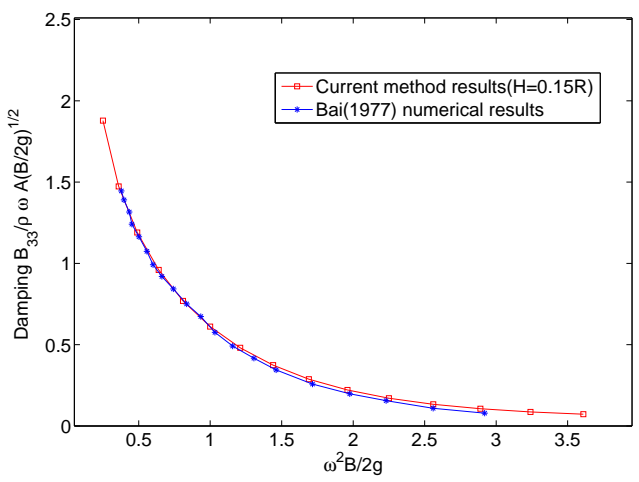

(b) Heave damping coefficient $B_{33}$

Figure 5: Comparison of the predicted heave added mass coefficient $A_{33}$ (a) and damping coefficient $B_{33}$ (b) between the current method and the numerical results of Bai (1977) for circular a cylinder oscillating in heave motion in water depth $H=1.5 R$.

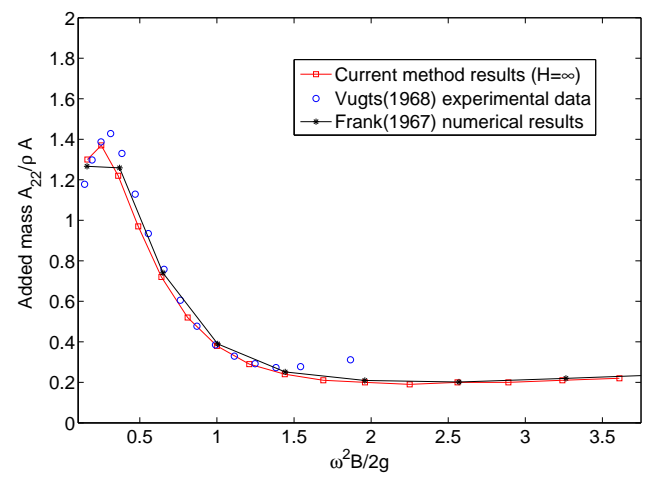

(a) Sway added mass coefficient $A_{22}$

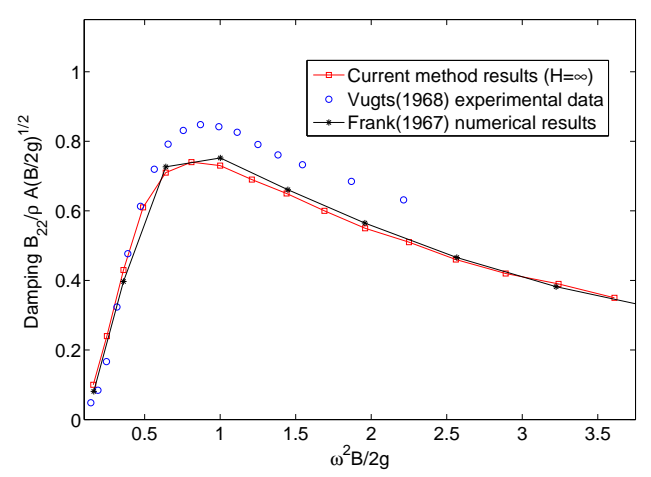

(b) Sway damping coefficient $B_{22}$

Figure 6: Comparison of the predicted sway added mass coefficient $A_{22}$ (a) and damping coefficient $B_{22}$ (b) between the current method, the experimental data of Vugts (1968) and numerical results of Frank (1967) for a circular cylinder oscillating in sway motion in water depth $H=\infty$.

damping coefficient $B_{33}$ decrease with increasing value of minor axis. These hydrodynamic coefficients are largest for the shallowest flat seabed condition. The added mass coefficient $A_{33}$ exhibits increasing values with similar trends observed over all frequencies examined whereas the damping coefficient $B_{33}$ converges to similar values as the frequency increases to $\omega^{2} B / 2 g=4.0$ for different seabed conditions. Figure 14 presents the time record of vertical force $F(t)$ excited on this cylinder when frequency $\omega^{2} B / 2 g=1.0$. The amplitudes of force $F(t)$ decrease as the value of minor axis increases. This is consistent with the frequency domain results as shown in Figures 12 and 13. There is no phase shift 


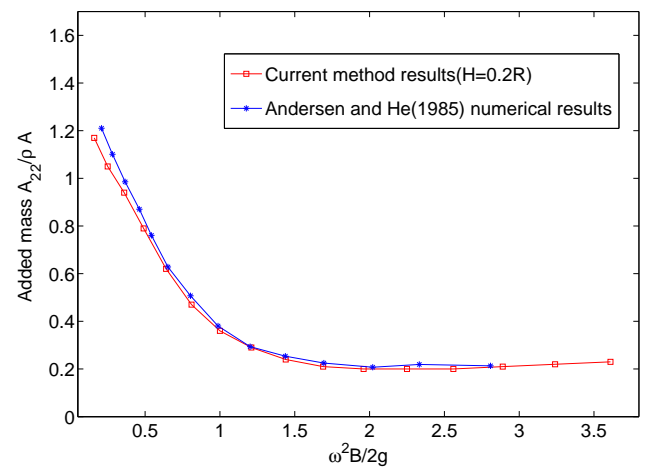

(a) Sway added mass coefficient $A_{22}$

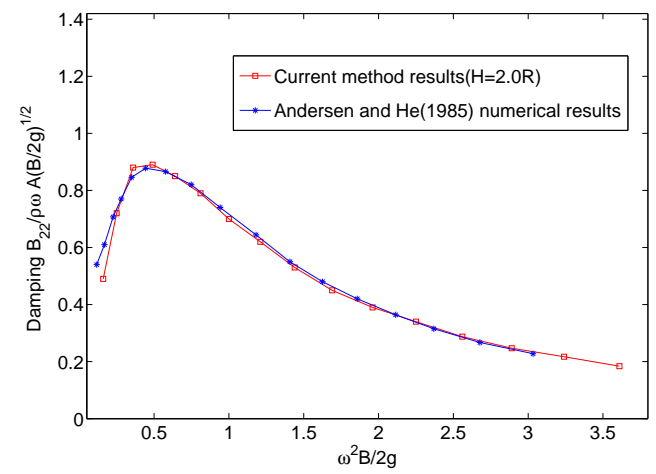

(b) Sway damping coefficient $B_{22}$

Figure 7: Comparison of the predicted sway added mass coefficient $A_{22}$ (a) and damping coefficient $B_{22}$ (b) between the current method and numerical results of Andersen and $\mathrm{He}$ (1985) for a circular cylinder oscillating in sway motion in water depth $H=2 R$.

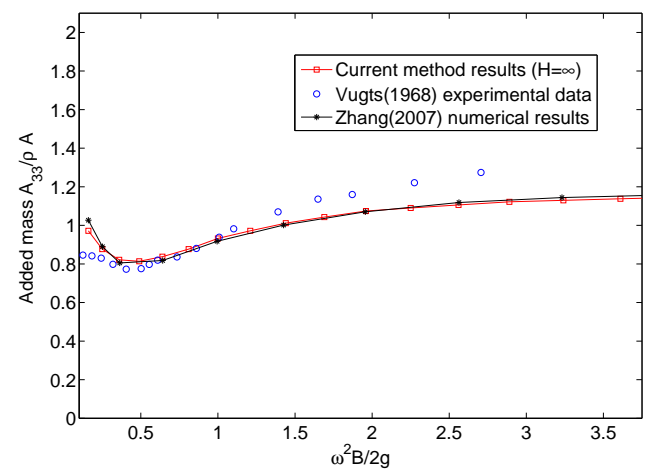

(a) Heave added mass coefficient $A_{33}$

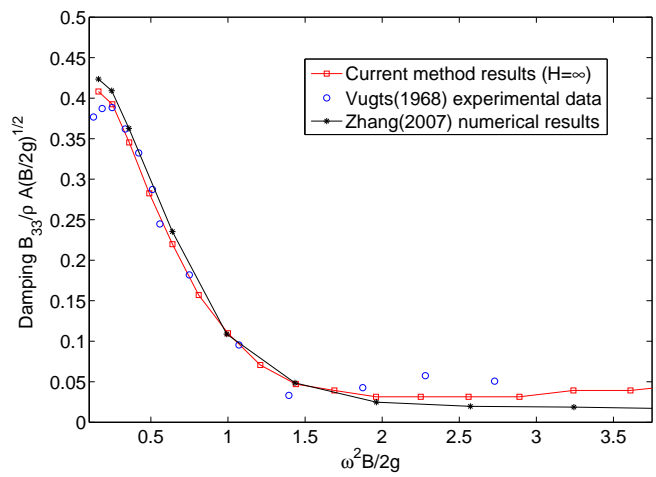

(b) Heave damping coefficient $B_{33}$

Figure 8: Comparison of the predicted heave added mass coefficient $A_{33}$ (a) and damping coefficient $B_{33}$ (b) between the current method the experimental data of Vugts (1968) and numerical results of Zhang and Beck (2007) for an oscillating rectangular cylinder in water depth $\mathrm{H}=\infty$.

observed in the force profile between a flat seabed and uneven conditions.

For this cylinder experiencing forced oscillatory sway motion above the different shaped seabeds, the hydrodynamic coefficients $A_{22}$ and $B_{22}$ are presented in Figures 15 and 16. Similar to the heave motion results, the sway motion added mass coefficients $A_{22}$ and damping coefficients $B_{22}$ increase with increasing value of minor axis. However the hydrodynamic effects are much smaller for sway motion than for heave motion and this is especially true when frequency $\omega^{2} B / 2 g$ is larger than 1.5 indicating the bottom topography has little influence. This implies that the various chosen shaped seabeds have little hydrodynamic 


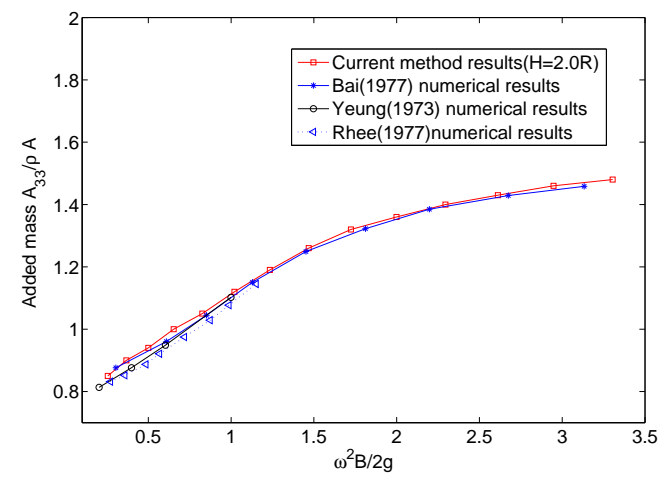

(a) Heave added mass coefficient $A_{33}$

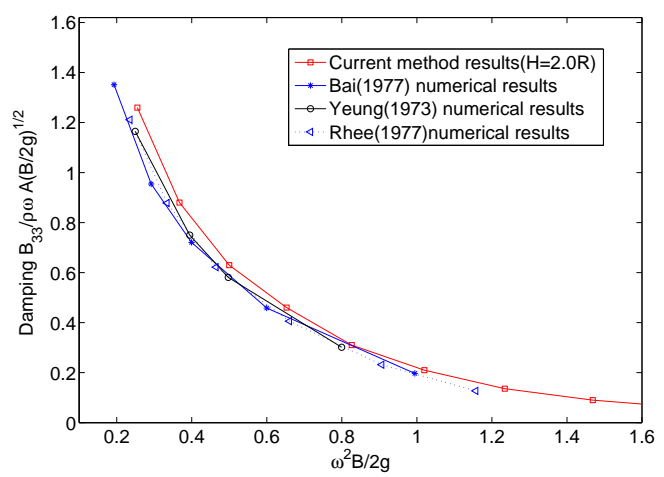

(b) Heave damping coefficient $B_{33}$

Figure 9: Comparison of the predicted heave added mass coefficient $A_{33}$ (a) and damping coefficient $B_{33}$ (b) between the current method, the numerical results of Bai (1977), and the numerical predictions of Yeung (1973) and Rhee (1977) for an oscillating rectangular cylinder in water depth $H=2 R$.

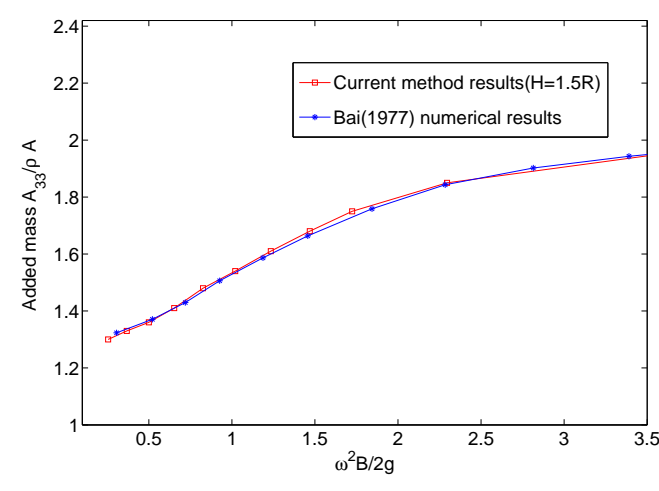

(a) Heave added mass coefficient $A_{33}$

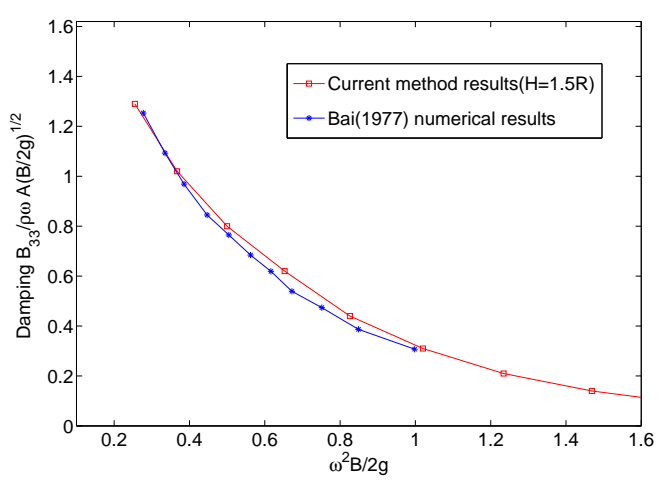

(b) Heave damping coefficient $B_{33}$

Figure 10: Comparison of the predicted heave added mass coefficient $A_{33}$ (a) and damping coefficient $B_{33}$ (b) between the current method and numerical predictions of Bai (1977) for an oscillating rectangular cylinder in water depth $H=1.5 R$.

influence on the wave-body interaction problems for sway motions when forced oscillatory frequency is larger than 1.5.

Figure 17 presents the time record of horizontal force $F(t)$ when frequency $\omega^{2} B / 2 g=1.0$. It is observed that the amplitude of $F(t)$ marginally increases as the values of the minor axis decrease. This can be explained by the fact that the fluid flow has a larger normal velocity near the seabed for heave motion than for sway motion and the velocity is reduced to zero at the seabed because of its presence. Therefore in the concave seabed cases the flow velocity changes more significantly for heave motion than for sway motion. 


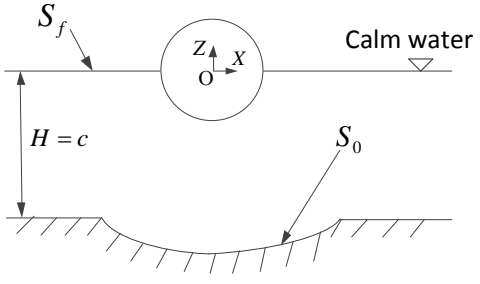

(a)

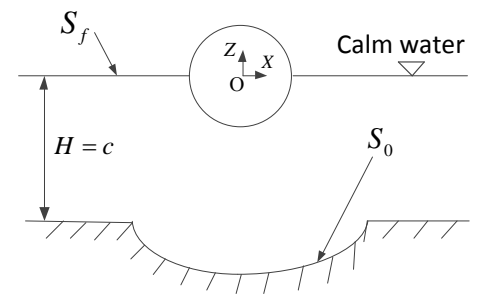

(b)

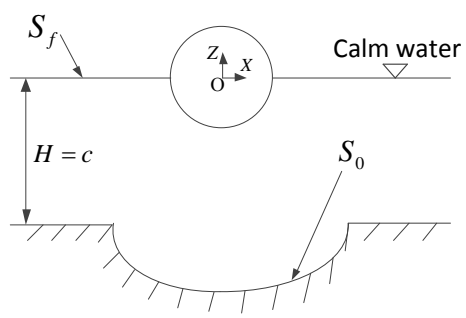

(c)

Figure 11: Sketches of wave-body interaction problem with three different seabed topographies of semi-elliptical concave shapes $(a),(b)$ and $(c)$.

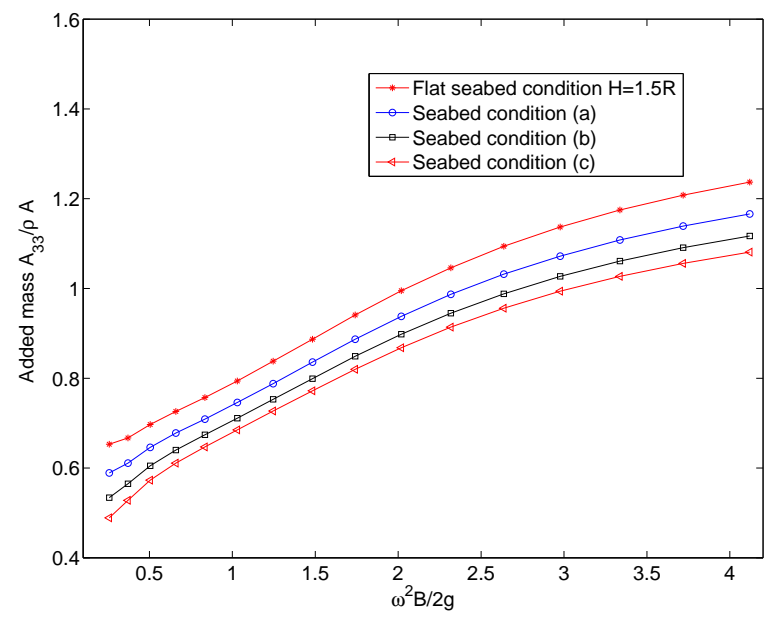

Figure 12: Comparison of heave added mass coefficient $A_{33}$ for a circular cylinder experiencing forced oscillatory heave motion above a flat seabed in water depth $H=1.5 R$, and uneven seabed shapes $(a),(b)$ and $(c)$ as shown in Figure 11. 


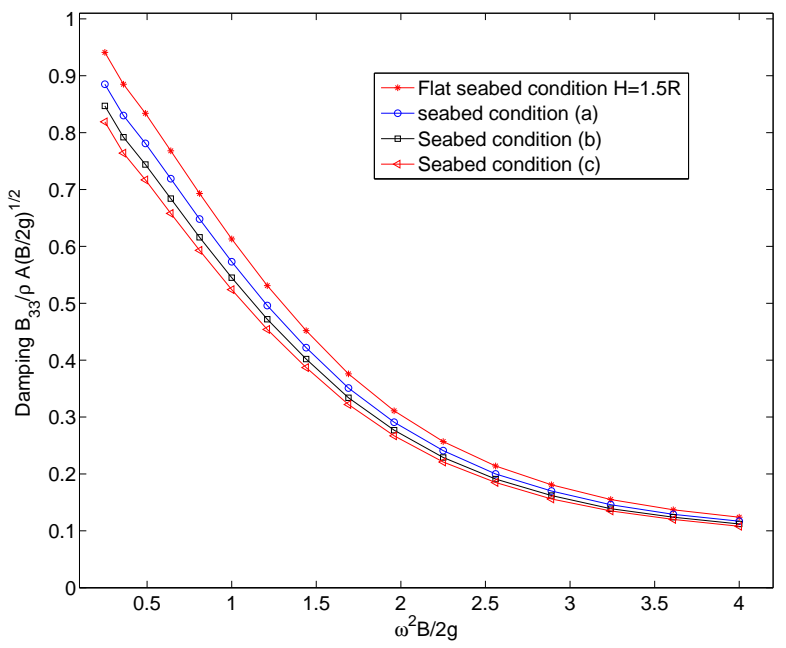

Figure 13: Comparison of heave damping coefficient $B_{33}$ for a circular cylinder experiencing forced oscillatory heave motion above a flat seabed in water depth $H=1.5 R$, and uneven seabed shapes $(a),(b)$ and $(c)$ as shown in Figure 11.

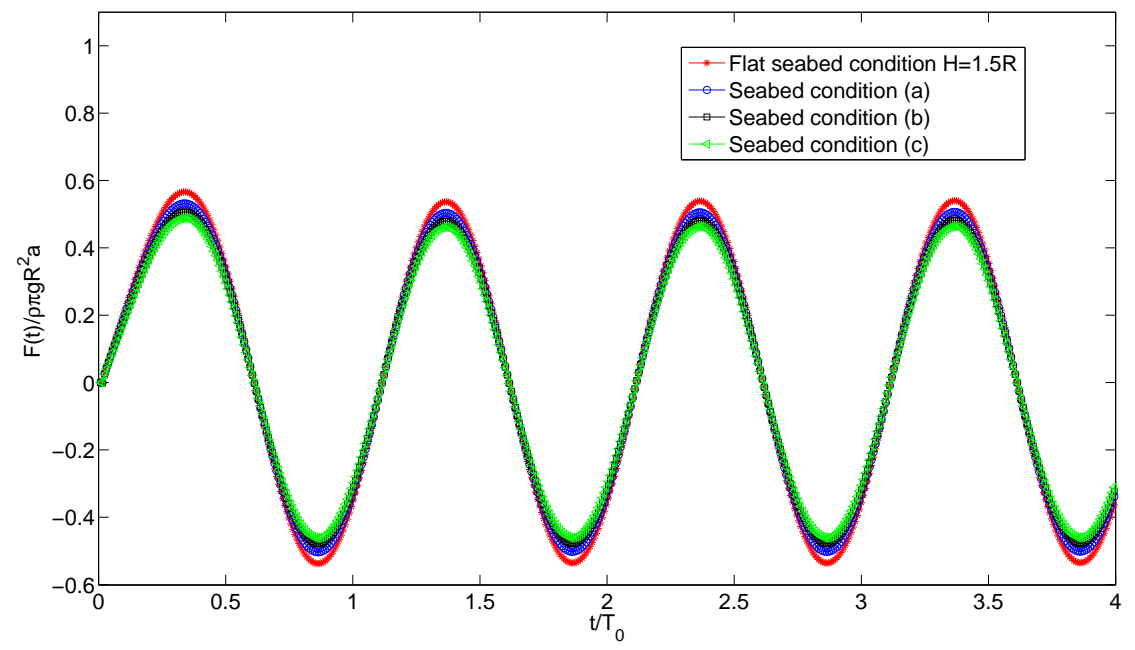

Figure 14: Time record of vertical force $F(t)$ for a circular cylinder experiencing forced oscillatory heave motion above a flat seabed in water depth $H=1.5 R$, and uneven seabed shapes $(a),(b)$ and $(c)$ as shown in Figure 11. 


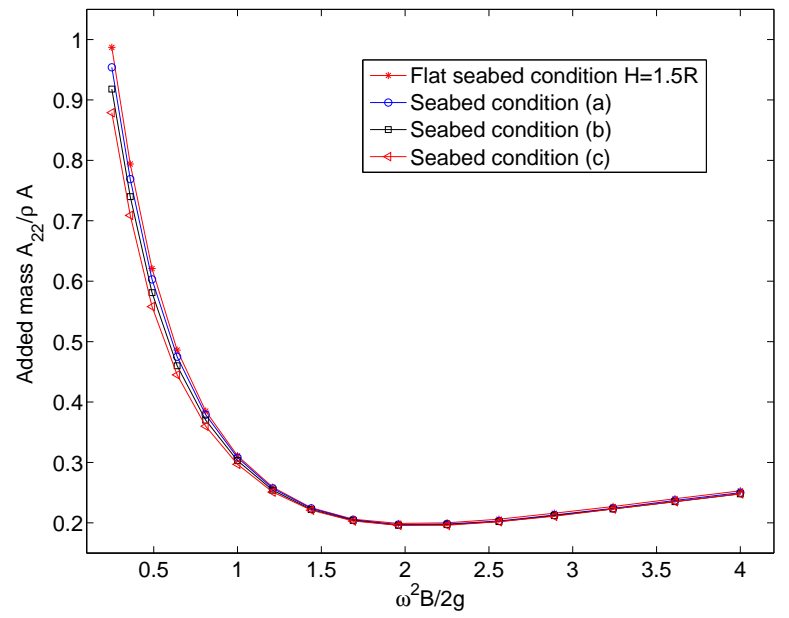

Figure 15: Comparison of sway added mass coefficient $A_{22}$ for a circular cylinder experiencing forced oscillatory sway motion above a flat seabed in water depth $H=1.5 R$, and uneven seabed shapes $(a),(b)$ and $(c)$ as shown in Figure 11.

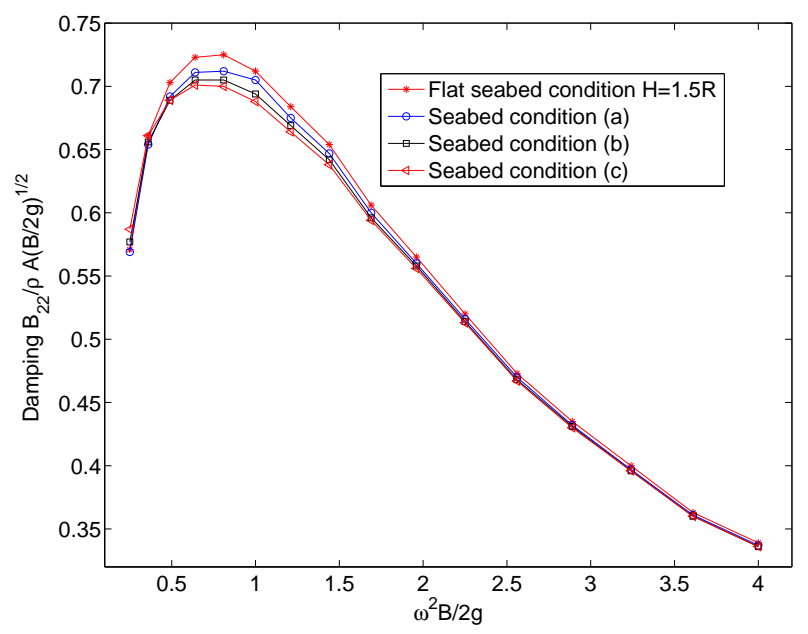

Figure 16: Comparison of sway damping coefficient $B_{22}$ for a circular cylinder experiencing forced oscillatory sway motion above a flat seabed in water depth $H=1.5 R$, and uneven seabed shapes $(a),(b)$ and $(c)$ as shown in Figure 11.

\subsection{Wave-body interaction problem involving a convex or humped shaped seabed}

The numerical simulation is further extended to a seabed at water depth $H=1.5 R$ exhibiting a concave bump of semi-elliptical shape over which the body oscillates as shown in Figure 18. The major axis $L m a j=2.0 R$ and the 


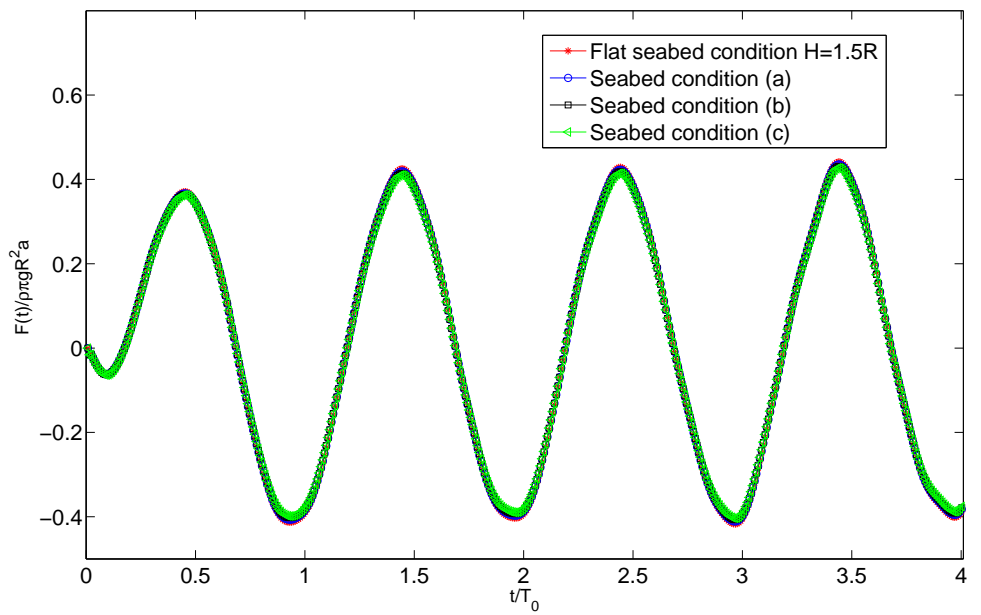

Figure 17: Time record of horizontal force $F(t)$ for a circular cylinder experiencing forced oscillatory sway motion above a flat seabed at water depth $H=1.5 R$, and uneven seabed shapes $(a),(b)$ and $(c)$ as shown in Figure 11.

minor axis $\operatorname{Lmin}=0.1 R$ or $\operatorname{Lmin}=0.3 R$ respectively indicated as $(d)$ and $(e)$ in Figure 18.

Figures 19 and 20 show comparisons of added mass coefficient $A_{33}$ and $B_{33}$ for this circular cylinder experiencing forced oscillatory heave motion above a flat seabed $H=1.5 R$ and uneven seabed topographies $(d)$ and $(e)$ as shown in Figure 18 . The added mass coefficient $A_{33}$ significantly increases in value over the frequency range with increasing value of minor axis. A similar phenomenon is observed for damping coefficient $B_{33}$. Figure 21 shows the time record of vertical force $F(t)$ excited on this cylinder when frequency $\omega^{2} B / 2 g=1.0$. This cylinder experiences the largest vertical force for the shallowest water depth seabed shape $(e)$ and smallest for the deeper flat seabed. This further demonstrates that the water depth beneath the floating body has a significant effect on wave-body interaction problems.

For this cylinder experiencing forced oscillatory sway motion above different seabed topographies, the hydrodynamic coefficients $A_{22}$ and $B_{22}$ are presented in Figures 22 and 23. Both these hydrodynamic coefficients increase with increasing value of minor axis. Figure 24 shows the time record of horizontal force $F(t)$ excited on this cylinder when frequency $\omega^{2} B / 2 g=1.0$. The cylinder experiences larger force amplitudes with increasing value of minor axis causing the water depth to decrease. Both these two hydrodynamic coefficients change in values more significantly for convex shaped seabeds than observed for concave seabed conditions, further emphasizing the role of water depth. This is because in the former shaped scenarios the average water depth is less than the latter cases, again indicating that there is an increased change of hydrodynamic 
characteristics as the water depths decrease. In a similar finding to the concave topographies, a seabed of convex shape has less influence on the hydrodynamic properties associated with sway than on heave motion. The reasoning is similar to the explanation given in the previous section for the concave seabed topographies.

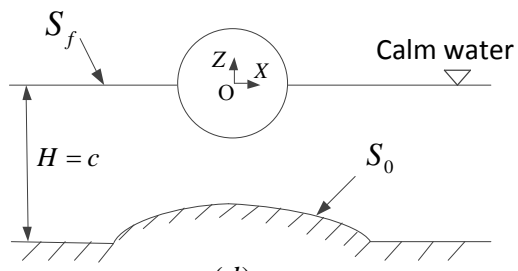

$(d)$

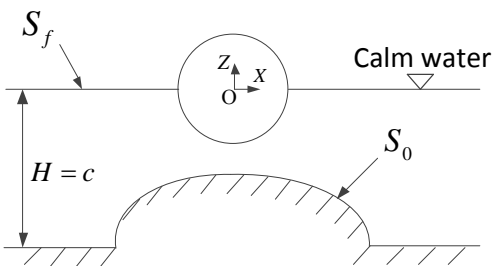

$(e)$

Figure 18: Sketches of wave-body interaction problem above a semi-elliptical bump or convex shaped seabed in different mean water depths when $(d) L_{m a j}=2.0 R, L_{m i n}=0.1 R$ and $(e)$ $L_{m a j}=2.0 R, \operatorname{Lmin}=0.3 R$.

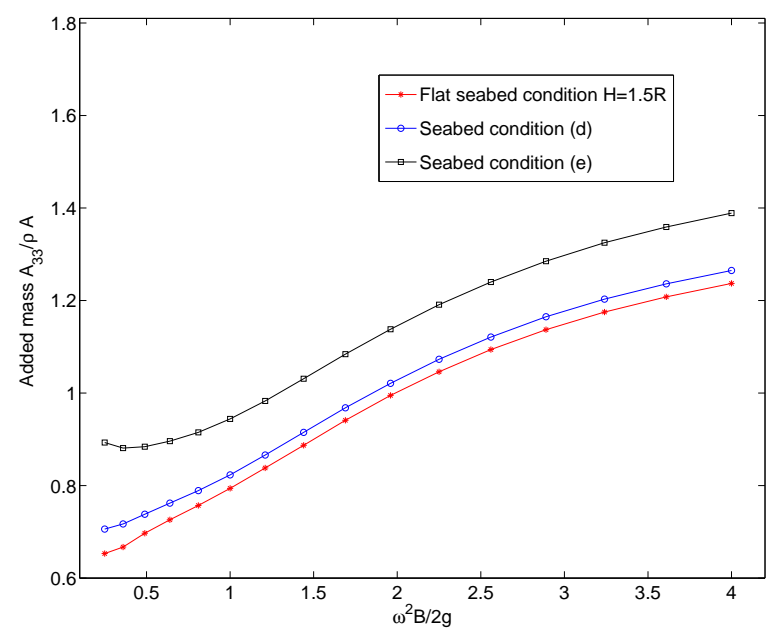

Figure 19: Comparison of heave added mass coefficient $A_{33}$ for a circular cylinder experiencing forced oscillatory heave motion above a flat seabed condition $H=1.5 R$, and uneven seabed shapes $(d)$ and $(e)$ as shown in Figure 18.

\subsection{General discussion of seabed shape and water depth}

In this discussion, we examine the influence of water depth and modification of seabed surface profiles on hydrodynamic properties. This is achieved through 


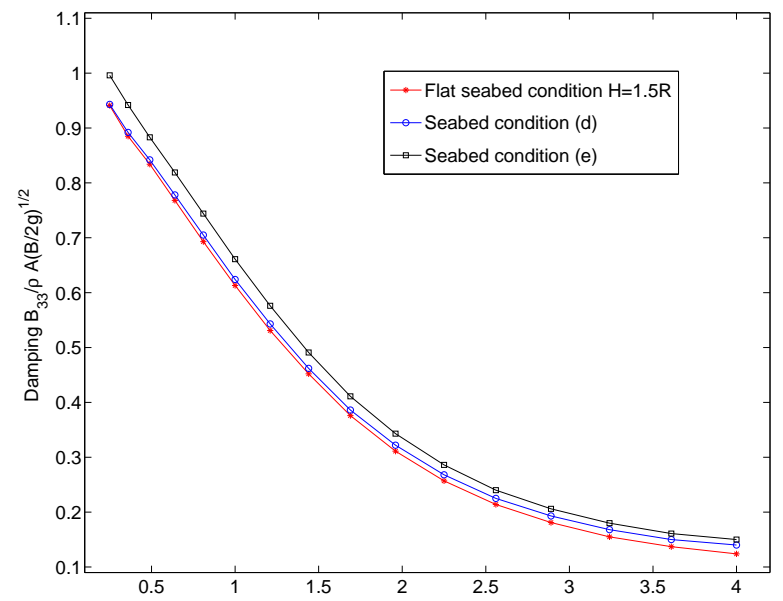

Figure 20: Comparison of heave damping coefficient $B_{33}$ for a circular cylinder experiencing forced oscillatory heave motion above a flat seabed $H=1.5 R$, and uneven seabed shapes $(d)$ and $(e)$ as shown in Figure 18.

an illustrative comparison of hydrodynamic characteristics associated with the circular cylinder in oscillatory heave motion above a flat seabed at depths $H=$ $1.2 R, H=1.5 R$ and $H=1.8 R$ as well as the semi-elliptical shapes shown in Figures 11 and 18 for, mainly, $L_{\text {min }}=0.3 R$. Similarly, the interim flat seabeds at $H=1.4 R$ and $H=1.6 R$ are the asymptotic topographies for water depth $H=1.5 R, L_{m a j}=\infty$ value, and $L_{m i n}=0.1 R$. By varying the value of $L_{m a j}$ allows a measure of verification of the mathematical model and numerical scheme of study because the findings for these interim states must lie between the asymptotic predictions of the flat seabed cases. Furthermore, for a fixed semiminor axis value, predictions of the hydrodynamic coefficients provide a measure of the influence of the size of the seabed obstacle or indentation through changing the length of the semi-major axis. Under these circumstances the maximum water depth remains constant, but changes to the value of the semi-major axis implies the mean water depth below the floating body alter influencing the values of the hydrodynamic coefficients.

Figures 25 and 26 compare the heave added mass coefficient $A_{33}$ and damping coefficient $B_{33}$ for the circular cylinder experiencing forced oscillatory heave motion above flat seabed conditions $H=1.8 R, H=1.4 R, H=1.2 R$ and uneven seabed conditions $(c),(d)$ and $(e)$ defined when $H=1.5 R$. Flat seabed condition $H=1.8 R$ is at the deepest point of case $(c)$ and flat seabed conditions $H=1.4 R$ and $H=1.2 R$ are corresponding to the shallowest point of case $(d)$ and $(e)$ respectively. The influence of uneven seabed shape on hydrodynamic characteristics becomes more obvious when the values of water depth decrease.

Figures 27 and 28 further compare the hydrodynamic coefficients for the 


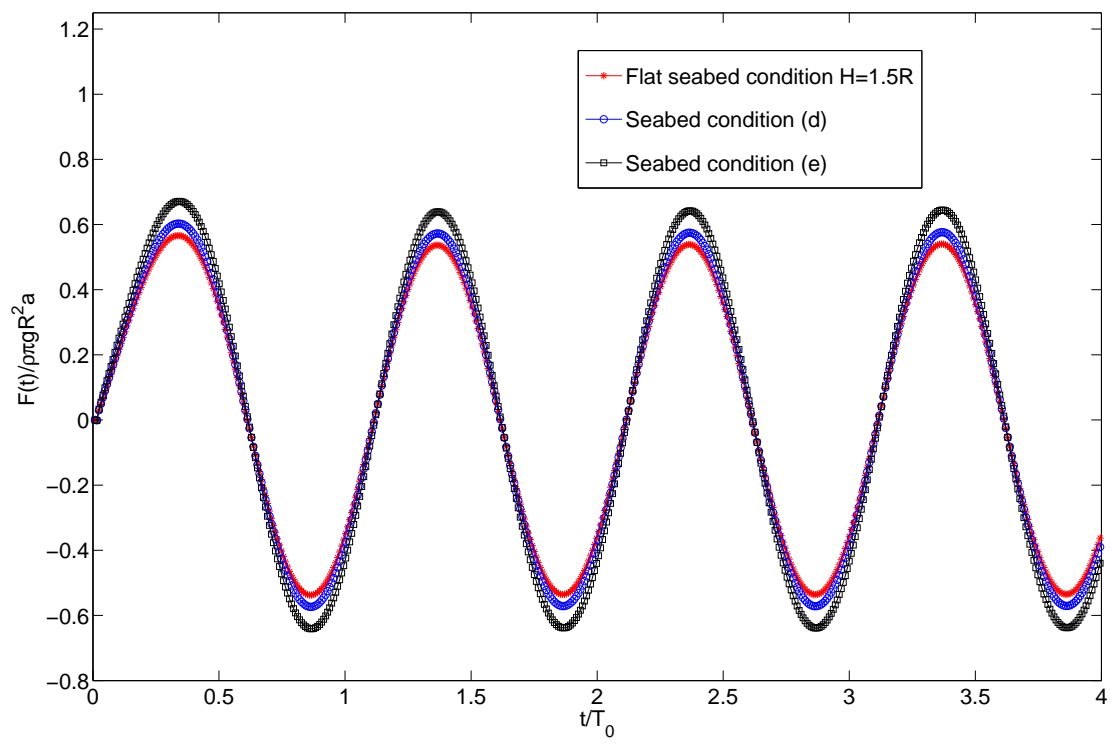

Figure 21: Time record of vertical force $F(t)$ for a circular cylinder experiencing forced oscillatory heave motion above a flat seabed $H=1.5 R$, and uneven seabed shapes $(d)$ and $(e)$ as shown in Figure 18.

circular cylinder experiencing forced heave oscillatory motion above flat seabeds at $H=1.2 R$ and $H=1.5 R$ and uneven seabed conditions $(e)$ at $H=1.5 R$ with varying $L_{m a j}=1.0 R, 2.0 R, 4.0 R, 8.0 R$ or $16.0 R$. It is noted that in all these cases, the minimum water depth below the floating body remains constant, $H=1.2 R$, but with increasing value of the semi-major axis $L_{m a j}$ the mean water depth reduces which is reflected in the comparative change of values of hydrodynamic coefficients between each value of $L_{m a j}$. The average water depth for seabed condition $(e)$ with $L_{m a j}=1.0$ is nearest to the flat seabed condition $H=1.5 R$ amongst the uneven cases. The results for seabed condition $(e)$ for $H=1.5 R$ with $L_{m a j}=16.0 R$ and $L_{m i n}=0.3 R$ closely approach the predictions for those associated with the flat seabed at $H=1.2 R$, as would be expected.

In the course of this investigation, the sensitivity of the mathematical model and convergence of solution were examined through computations involving different discretisation schemes. The stability of the numerical scheme was found robust producing solutions behaving in the expected appropriate manner as shown in this section. The consistent sets of predictions shown demonstrate that with minor modifications to the mathematical model and discretisation process, the approach described herein is capable of investigating body fluid interaction problems involving finite water depths and sloping or irregular shaped seabed profiles. 


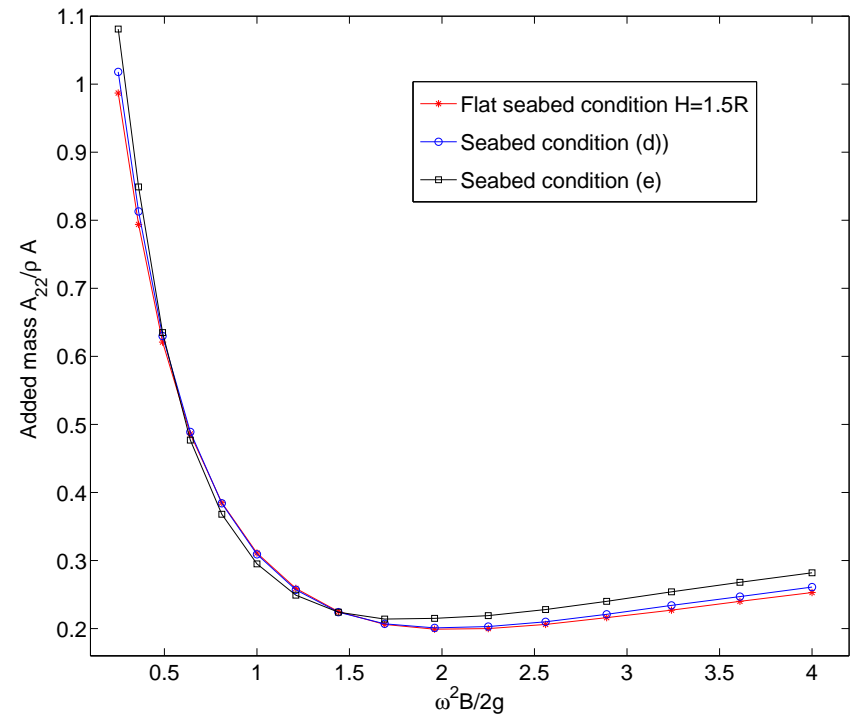

Figure 22: Comparison of sway added mass coefficient $B_{22}$ for a circular cylinder experiencing forced oscillatory sway motion above a flat seabed $H=1.5 R$, and uneven seabed shapes $(d)$ and $(e)$ as shown in Figure 18.

Naturally, this idealized mathematical model needs re-examination when forward speed of the body is introduced especially in the shallowest depths when the oscillating body experiences sinkage and trim and the free surface disturbance may be of a more complex form. Furthermore, the seabed is treated as a solid but, in reality, sedimentation occurs and the mathematical model requires a description of this changing environment. 


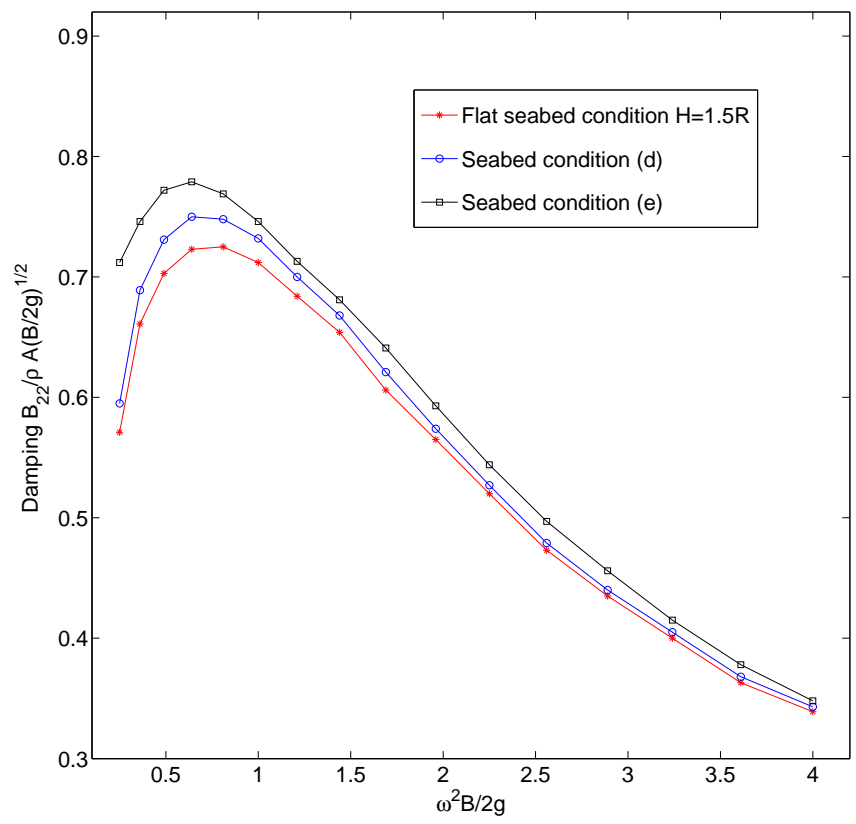

Figure 23: Comparison of sway damping coefficient $B_{22}$ for a circular cylinder experiencing forced oscillatory sway motion above a flat seabed $H=1.5 R$, and uneven seabed shapes $(d)$ and $(e)$ as shown in Figure 18. 


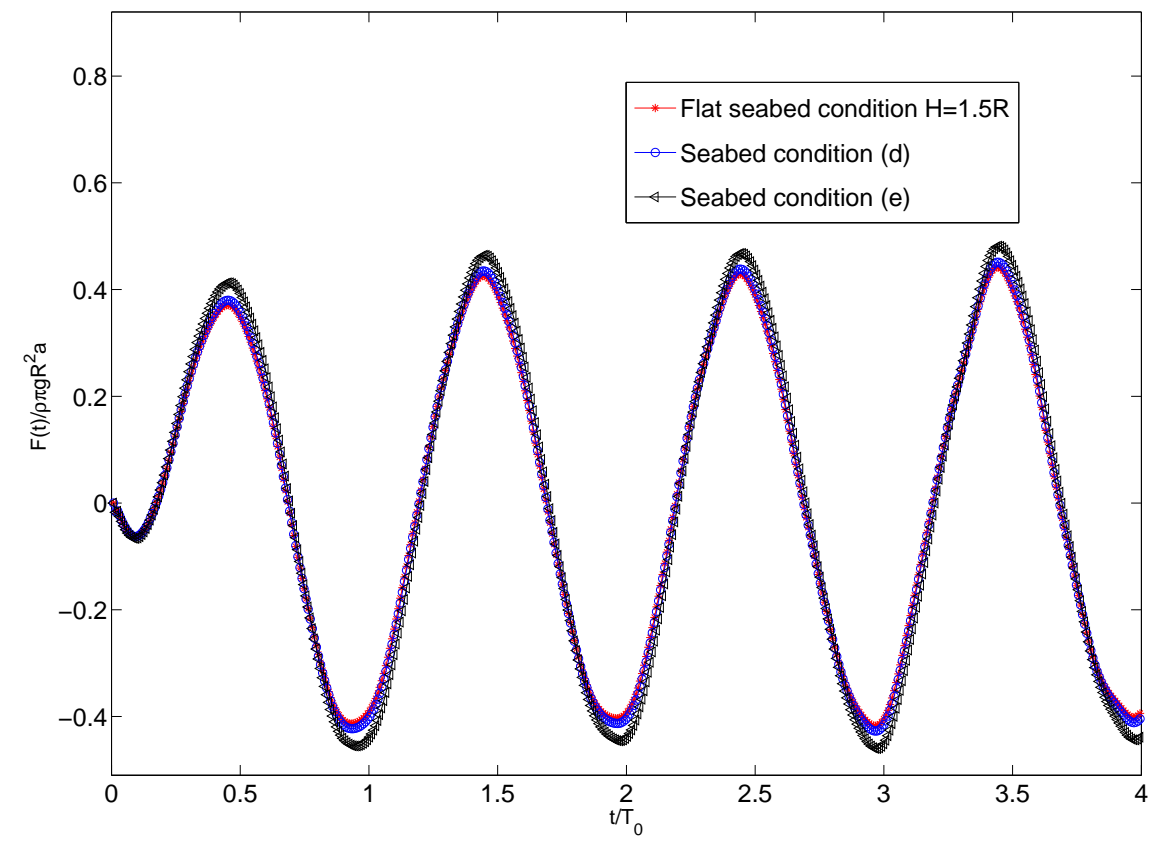

Figure 24: Time record of horizontal force $F(t)$ for a circular cylinder experiencing forced oscillatory sway motion above a flat seabed $H=1.5 R$, and uneven seabed shapes $(d)$ and $(e)$ as shown in Figure 18. 


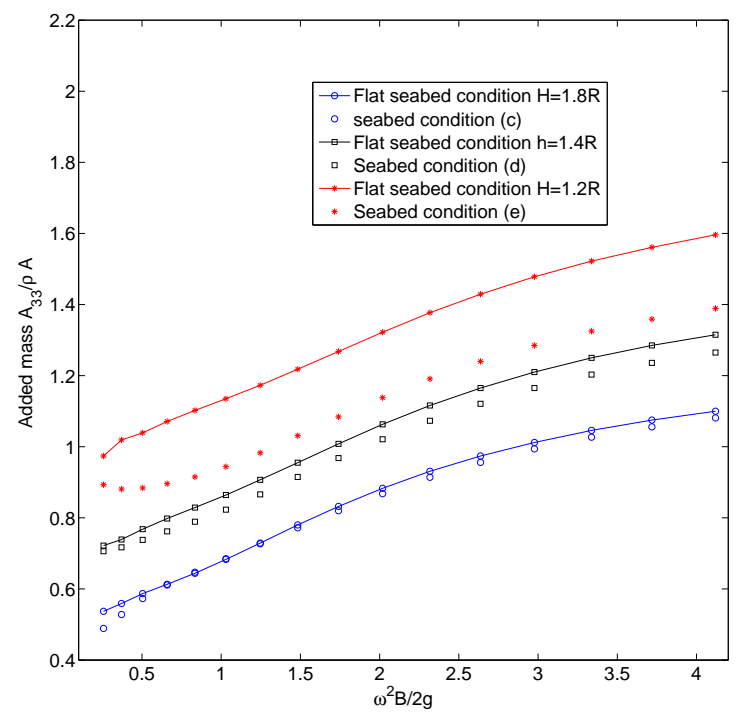

Figure 25: Comparison of heave added mass coefficient $A_{33}$ for a circular cylinder experiencing forced oscillatory heave motion above flat seabed $H=1.8 R, H=1.4 R, H=1.2 R$ and uneven seabed shape $(c)$ as shown in Figure 11, and $(d)$ and $(e)$ as shown in Figure 18 at $H=1.5 R$.

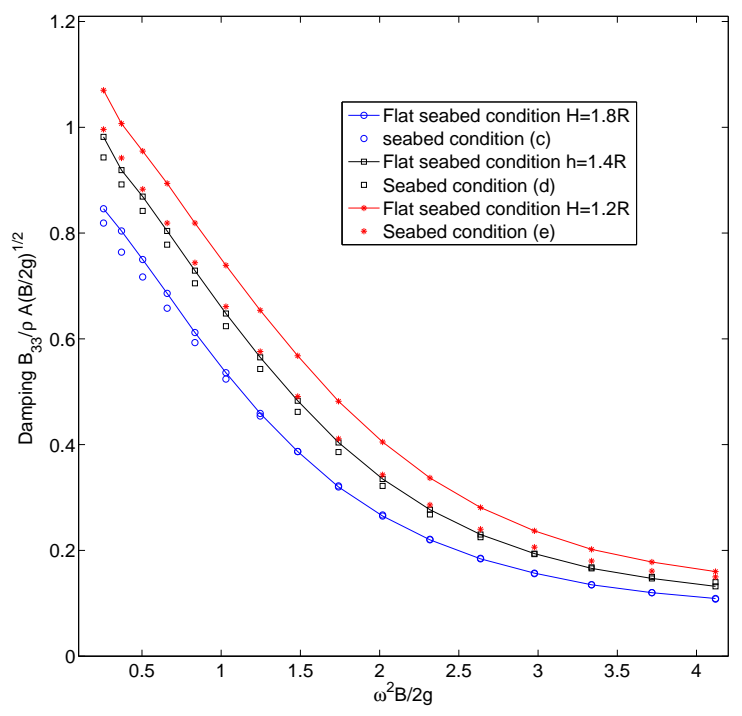

Figure 26: Comparison of heave damping coefficient $B_{33}$ for a circular cylinder experiencing forced oscillatory heave motion above a flat seabed $H=1.8 R, H=1.4 R, \mathrm{H}=1.2 R$ and uneven seabed shape $(c)$ as shown in Figure 11 and $(d)$ and $(e)$ as shown in Figure 18 at $H=1.5 R$. 


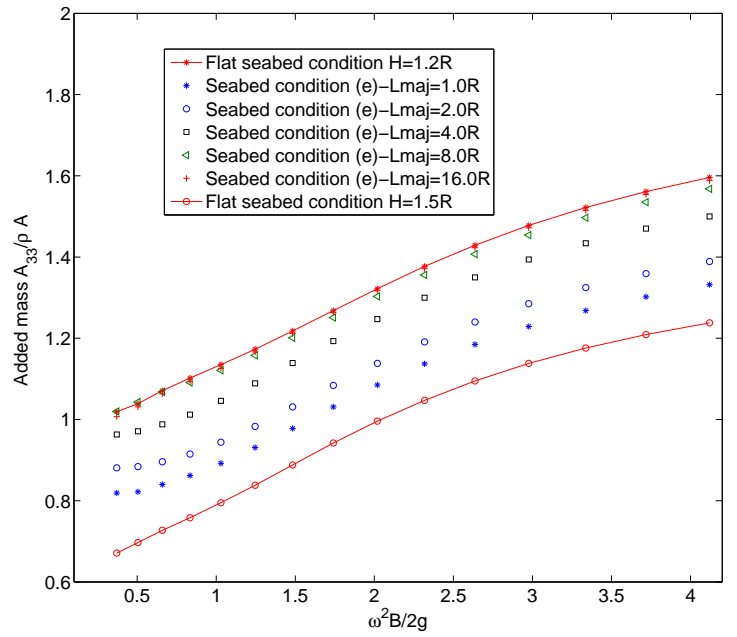

Figure 27: Comparison of heave added mass coefficient $A_{33}$ for a circular cylinder experiencing forced oscillatory heave motion above a flat seabed $H=1.2 R$ and $H=1.5 R$ and uneven seabed shape $(e)$ as shown in Figure 18 at $H=1.5 R$ with $L m a j=1.0 R, 2.0 R, 4.0 R, 8.0 R, 16.0 R$.

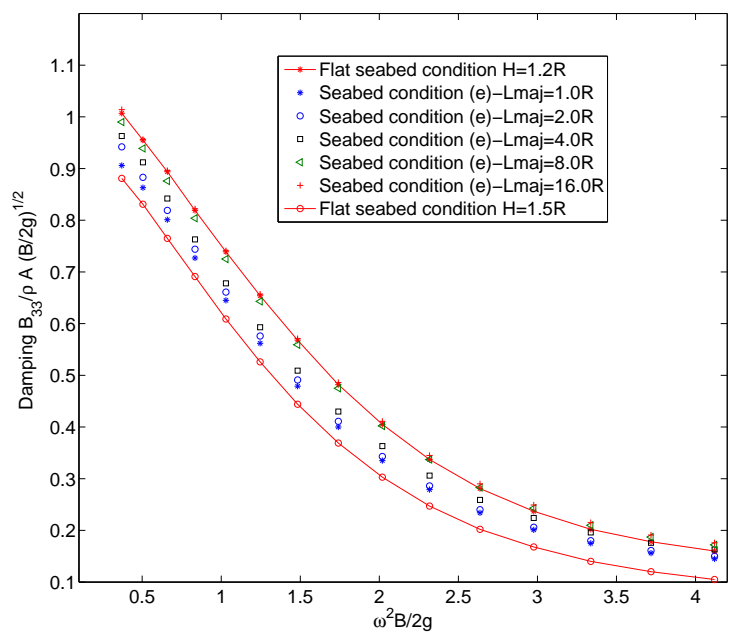

Figure 28: Comparison of heave damping coefficient $B_{33}$ for a circular cylinder experiencing forced oscillatory heave motion above flat seabed $H=1.2 R$ and $H=1.5 R$ and uneven seabed shape $(e)$ as shown in Figure 18 at $H=1.5 R$ with $L m a j=1.0 R, 2.0 R, 4.0 R, 8.0 R, 16.0 R$. 


\section{Conclusions}

A continuous Rankine source panel method is adopted to study a twodimensional body experiencing forced oscillatory heave or sway motion above various seabed seabed topographies. The source panels are placed exactly on the free and seabed surfaces. No desingularized technique is required and therefore numerical errors arising from this approximation technique are excluded. The space increment source distribution method developed in our previous research for deep water, wave-body interaction problems is extended to arbitrary depth situations. Both these types of problems can be investigated within one mathematical model framework without the necessity to introduce new numerical techniques. The developed numerical model accommodates flat and various uneven seabed topographies and this demonstrates the wide applicability of this method for different boundary condition problems.

Numerical simulations are performed extensively for circular and rectangular cylinders experiencing both heave and sway motions. The proposed method demonstrates favourable agreement with published data when the body oscillates in deep or finite water depths above a flat seabed. Numerical simulations show that a seabed displaying concave or bump/convex, semi-elliptical shapes have a larger influence on the hydrodynamic properties associated with heave motion than sway motion. For example, with increasing size of the minor axis of the semi-elliptic shape the predicted added mass and damping coefficients significantly change in magnitudes when the body undergoes forced oscillatory heave motion because of the changes to the mean water depth. This study confirms that the form of the shape of the seabed directly affects the water depth or, more crudely, its mean value below the floating body. It is this parameter which significantly influences the hydrodynamic characteristics of the floating body.

In this study, it is assumed that the forced amplitude of oscillatory motion is small compared to the bodys dimensions with the linearized free surface and body boundary conditions satisfied on the mean wetted surfaces. In our previous study the continuous Rankine source method coupled to an Euler-Lagrange algorithm was developed successfully to solve nonlinear body boundary problems in deep water. However, the mathematical approach developed in that study adopted a desingularized method whereas the current investigation improves and extends the continuous Rankine source concept within the family of singularized methods. In principle, a similar Euler- Lagrange algorithm can be integrated into the discussed numerical method described herein to study non-linear wave problems, which will be the focus of future studies.

\section{Acknowledgements}

The authors gratefully acknowledge the financial support provided by the Singapore Maritime Institute (SMI) Grant R-302-000-107-592. This work was partially supported by the 973 program of China (grant No.2013CB036103) and the National Naturel Science Foundation of China (grant No. 11571240). 


\section{References}

Andersen, P., He, W., 1985. On the calculation of two-dimensional added mass and damping coefficients by simple Green's function technique. Ocean Engineering 12, 425-451.

Bai, K., 1977. The added mass of two-dimensional cylinders heaving in water of finite depth. Journal of Fluid Mechanics 81, 85-105.

Bandyk, P., 2009. A body-exact strip theory approach to ship motion computations. Ph.D. thesis. University of Michigan.

Bandyk, P., Beck, R., 2011. The acceleration potential in fluid-body interaction problems. Journal of Engineering Mathematics 70, 147-163.

Beck, R., Cao, Y., Lee, T., 1994. Fully nonlinear water wave computations using the desingularized method, in: Proceedings of the 6th International Conference on Numerical Ship Hydrodynamics, pp. 3-20.

Beck, R., Scorpio, S., 1995. A desingularized boundary integral method for fully nonlinear water wave problems, in: Proceedings of the 12th Australasian Fluid Mechanics Conference, pp. 255-258.

Buchner, B., 2006. The motions of a ship on a sloped seabed, in: Proceedings of the 25th International Conference on Offshore Mechanics and Arctic Engineering, pp. 339-347.

Cao, Y., 1991. Computation of nonlinear gravity waves by a desingularized boundary integral method. Ph.D. thesis. University of Michigan.

Cao, Y., Schultz, W., Beck, R., 1991a. Three-dimensional desingularized boundary integral methods for potential problems. International Journal for $\mathrm{Nu}-$ merical Methods in Fluids 12, 785-803.

Cao, Y., Schultz, W., Beck, R., 1991b. Two-dimensional solitary waves generated by a moving disturbance, in: Proceedings of the 6th International Workshop on Water Waves and Floating Bodies, pp. 25-28.

Chen, Z.M., 2012. A vortex based panel method for potential flow simulation around a hydrofoil. Journal of Fluids and Structures 28, 378-391.

De Hauteclocque, G., Rezende, F., Giorgiutti, Y., Chen, X.B., 2009. Wave kinematics and seakeeping calculation with varying bathymetry, in: Proceedings of the 28th International Conference on Ocean, Offshore and Arctic Engineering, pp. 515-523.

Faltinsen, O., 1993. Sea loads on ships and offshore structures. Cambridge university press. 
Feng, A., 2014. Numerical simulation of nonlinear wave-body problem based on desingularized Rankine Source and mixed Euler-Lagrange method. Ph.D. thesis. University of Southampton.

Feng, A., Chen, Z.M., Price, W., 2014. A Rankine source computation for three dimensional wave-body interactions adopting a nonlinear body boundary condition. Applied Ocean Research 47, 313-321.

Feng, A., Chen, Z.M., Price, W., 2015a. A continuous desingularized source distribution method describing wave-body interactions of a large amplitude oscillatory body. Journal of Offshore Mechanics and Arctic Engineering 137.

Feng, A., Chen, Z.M., Price, W., 2015b. A desingularized rankine source method for nonlinear wave-body interaction problems. Ocean Engineering 101, 131141.

Ferreira, M., Newman, J., 2009. Diffraction effects and ship motions on an artificial seabed, in: Proceedings of the 24th International Workshop on Water Waves and Floating Bodies.

Finn, P., 2003. Large amplitude nonlinear seakeeping using a desingularized method. Ph.D. thesis. University of Michigan.

Frank, W., 1967. Oscillation of cylinders in or below the free surface of deep fluids. Technical Report. David W. Taylor Naval Ship Research Development Center.

Huang, Y., 1997. Nonlinear ship motions by a Rankine panel method. Ph.D. thesis. Massachusetts Institute of Technology.

Kim, C., 1969. Calculation of hydrodynamic forces for cylinders oscillating in shallow water. Journal of Ship Research 13, 137-164.

Kim, C., 1975. Effect of mesh size on the accuracy of finite-water added mass. Journal of Hydronautics 9, 125-126.

Kring, D.C., 1994. Time domain ship motions by a three-dimensional Rankine panel method. Ph.D. thesis. Massachusetts Institute of Technology.

Lamb, H., 1945. Hydrodynamics. 6th edition, Dover Publications, Inc, New York, pp.59-60.

Lee, C., 1995. WAMIT Theory Manual, MIT Report 95-2. Technical Report. Department of Ocean Engineering, MIT.

Lee, T., 1992. Nonlinear radiation problems for a surface-piercing body. Ph.D. thesis. University of Michigan.

Lee, T., 2003. Fully nonlinear wave computations for arbitrary floating bodies using the delta method. Journal of Hydrodynamics 15, 24-31. 
Newman, J., 2012. Scattering by a cylinder with variable bathymetry, in: Proceedings of the 27th International Workshop on Water Waves and Floating Bodies.

Rhee, K., 1977. 2-dimensional hydrodynamic forces of heaving, swaying and rolling cylinders on a free surface of a water of finite depth. Journal of the Society of Naval Architects of Korea , 13-22.

Schultz, W., Cao, Y., Beck, R., 1990. Three-dimensional nonlinear wave computation by desingularized boundary integral method, in: Proceedings of the 5th International Workshop on Water Waves and Floating Bodies, pp. 169-172.

Scorpio, S., 1997. Fully nonlinear ship-wave computations using a multipole accelerated, desingularized method. Ph.D. thesis. University of Michigan.

$\mathrm{Su}$, D.T., Liu, T.L., Ou, C.H., 2008. Numerical investigation into effects of seabed topography on flows in and around artificial reefs. Fisheries Science $74,236-254$.

Van Oortmerssen, G., 1976. The motions of a ship in swallow water. Ocean Engineering 3, 221-255.

Vugts, J., 1968. The hydrodynamic coefficients for swaying, heaving and rolling cylinders in a free surface. International Shipbuilding Progress 15, 251-275.

Yeung, R., 1981. Added mass and damping of a vertical cylinder in finite-depth waters. Applied Ocean Research 3, 119-133.

Yeung, R.W.C., 1973. A singularity-distribution method for free-surface flow problems with an oscillating body. Ph.D. thesis. University of California, Berkeley.

Yu, Y., Ursell, F., 1961. Surface waves generated by an oscillating circular cylinder on water of finite depth: theory and experiment. Journal of Fluid Mechanics 11, 529-551.

Zhang, X., Bandyk, P., Beck, R., 2007. Large amplitude body motion computations in the time-domain, in: Proceedings of the 9th International Conference on Numerical Ship Hydrodynamics.

Zhang, X., Bandyk, P., Beck, R., 2010. Time-domain simulations of radiation and diffraction forces. Journal of Ship Research 54, 79-94.

Zhang, X., Beck, R., 2007. Computations for large-amplitude two-dimensional body motions. Journal of Engineering Mathematics 58, 177-189.

Zhang, X., Beck, R., 2008. Three-dimensional large amplitude body motions in waves. Journal of Offshore Mechanics and Arctic Engineering 130, 16-23. 